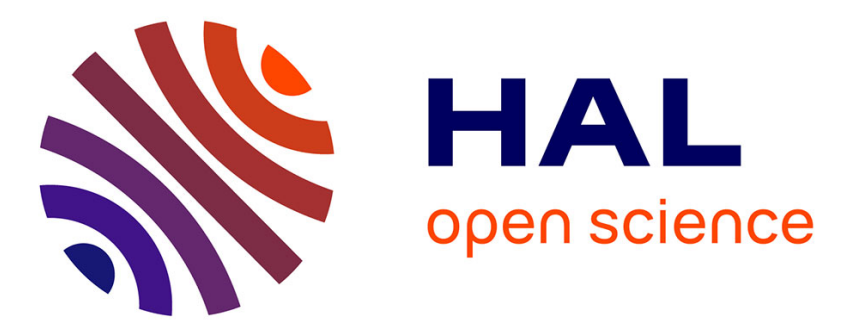

\title{
Estimation of local stresses and elastic properties of a mortar sample by FFT computation of fields on a 3D image
}

Julie Escoda, François Willot, Dominique Jeulin, Julien Sanahuja, Charles Toulemonde

\section{To cite this version:}

Julie Escoda, François Willot, Dominique Jeulin, Julien Sanahuja, Charles Toulemonde. Estimation of local stresses and elastic properties of a mortar sample by FFT computation of fields on a 3D image. Cement and Concrete Research, 2011, 41 (5), pp.542-556. 10.1016/j.cemconres.2011.02.003 . hal-00553658

\section{HAL Id: hal-00553658}

https://hal-mines-paristech.archives-ouvertes.fr/hal-00553658

Submitted on 7 Jan 2011

HAL is a multi-disciplinary open access archive for the deposit and dissemination of scientific research documents, whether they are published or not. The documents may come from teaching and research institutions in France or abroad, or from public or private research centers.
L'archive ouverte pluridisciplinaire HAL, est destinée au dépôt et à la diffusion de documents scientifiques de niveau recherche, publiés ou non, émanant des établissements d'enseignement et de recherche français ou étrangers, des laboratoires publics ou privés. 


\title{
Estimation of local stresses and elastic properties of a mortar sample by FFT computation of fields on a 3D image
}

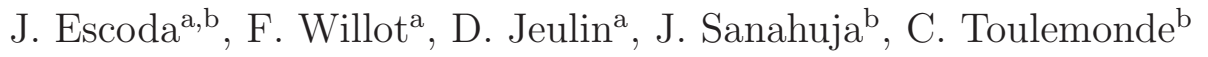 \\ ${ }^{a}$ Centre de Morphologie Mathématique, Mathématiques et Systèmes, Mines Paristech, \\ Fontainebleau, France \\ ${ }^{b}$ Département Matériaux et Mécanique des Composants, Électricité de France, \\ Moret-sur-Loing, France
}

\begin{abstract}
This study concerns the prediction of the elastic properties of a 3D mortar image, obtained by micro-tomography, using a combined image segmentation and numerical homogenization approach. The microstructure is obtained by segmentation of the 3D image into aggregates, voids and cement paste. Fullfields computations of the elastic response of mortar are undertaken using the Fast Fourier Transform method. Emphasis is made on highly-contrasted properties between aggregates and matrix, to anticipate needs for creep or damage computation. The Representative Volume Element, i.e. the volume size necessary to compute the effective properties with a prescribed accuracy, is given. Overall, the volumes used in this work were sufficient to estimate the effective response of mortar with a precision of $5 \%, 6 \%$ and $10 \%$ for contrasts ratio of 100, 1000 and 10000, respectively. Finally, a statistical and local characterization of the component of the stress field parallel to the applied loading is carried out.
\end{abstract}

Keywords: microstructure (B), elastic moduli (C), Fast Fourier Transform algorithm, image analysis (B), mortar (E)

2000 MSC: 74B05,

2000 MSC: $62 \mathrm{H} 35$,

2000 MSC: $74 \mathrm{~N} 15$

Preprint submitted to Cement and Concrete Research

January 7, 2011 


\section{Introduction}

The mechanical properties of cementitious and mortar-based composites strongly depend on the multi-scale distribution of fine aggregates and voids inside cement paste as well as on the shape and size of aggregates. Additionally, such materials usually exhibit a great diversity in the microstructure, as a consequence of their manufacturing process. Accordingly, a large number of experiments on real samples should be carried out to estimate precisely the properties of such composites. As a consequence, efforts have been made to simulate both the complex microstructures of such materials and their mechanical response, by numerical means. One of the main difficulties of the latter relates to large-size computations in homogenization. Memory and time-efficient numerical tools are required to predict the effective mechanical properties of microstructure samples. Microstructures are obtained either from a real material by segmentation of an image or from a random morphological simulation. Nagai et al [16] used a 3D concrete microstructure reconstructed from 2D successive sections to investigate both linear elastic behavior and cracks along the interface between aggregates and matrix. Microtomography has been used by Hain et al [9] to study linear elastic and viscoplastic behavior of hardened cement paste by the finite element method (FEM). Various random models of cementitious materials have been developed as well. For mortar and cement material, the model of Bentz [2], which takes into account hydration process, has been used by Hain et al [9], Haecker et al [7] and Bernard et al [3] to investigate linear elasticity of cement paste $[9,7]$ and of mortar [3]. Šmilauer et al [21] also used Bentz model combined with Fast-Fourier Transform method to study viscoelastic behavior of cement paste. They considered random microstructures of various sizes, the largest containing $200 \times 200 \times 200$ voxels, and low Young modulus contrast between matrix and aggregates. Bary et al [1] modeled cement paste as inclusions and pores embedded in the C-S-H matrix. Two models were considered for the particles: spheres or a mix of inclusions with spherical and prismatic shapes. The linear elastic response is evaluated thanks to FEM computations, for various types of boundary conditions. The effective properties are compared with the Mori-Tanaka and self-consistent analytical theories. Wriggers et al [24] proposed a random model of concrete made of spherical aggregates in a mortar matrix. The model assumes isotropy of the material and a minimal distance between particles, which depends on their volume fraction. The linear elastic response, as well as damage degradation, is investigated numer- 
ically usually with FEM. Two models of microstructures were also proposed by Häfner et al [8] and Caballero et al [5] and used to investigate the mechanical properties of concrete. In Häfner's model, the aggregates shape is a modified ellipsoid with a sine function added, with linear elasticity investigated with a multigrid method. Caballero et al [5] modeled concrete as large aggregates embedded in a matrix representing mortar and fine aggregates. Larger particles were generated as polyhedra obtained by Voronoï tessellation. An interface transition zone was taken into account with zero thickness element to investigate cracking. This model was used to compute numerically the response of mortar to a uniaxial tension test.

In the present work, a 3D image from microtomography is used to obtain the microstructure by segmentation. The strain and stress fields are computed using the Fast Fourier transform algorithm for various levels of contrast of properties between the aggregates and matrix Young moduli. The effective properties and representative volume element are derived from the FFT fullfield computations. Finally, a statistical study of the stress field is carried out, using the stress field histograms. A local characterization of high-stress zones in mortar is proposed, using morphological tools. It should be noted that the local characterization of fields, made possible by numerical computations, is a novel approach. It has been recently investigated by Rollett et al [17] for polycristals with viscoplastic behavior, using the FFT method, especially the distance between stress "hot-spots" and grains boundary.

\section{3D segmentation of the microstructure from microtomography}

\subsection{Mortar material}

A mortar sample made of CEM I 52.5 R CE NF Portland cement from Couvrot plant (cement of specific surface area of $3800 \mathrm{~cm}^{2} / \mathrm{g}$ ) and of nonreactive aggregates (limestone from Boulonnais quarries) is considered in this study. Mortar was prepared at a water to cement ratio of 0.6 and at the following composition: $537.8 \mathrm{~kg} / \mathrm{m}^{3}, 1614 \mathrm{~kg} / \mathrm{m}^{3}$ and $323 \mathrm{~kg} / \mathrm{m}^{3}$, for cement, fine aggregates and water respectively. A mold of size $40 \times 40 \times 160 \mathrm{~mm}$ was used. Three days after, the sample was wrapped into an aluminum foil and kept in a closed box during four days. It was finally steam-cured at $38{ }^{\circ} \mathrm{C}$. The studied sample is five years old. 


\subsection{Microstructure}

The mechanical behavior of a piece of mortar of size about $2.5^{3} \mathrm{~cm}^{3}$ is investigated. A 3D grayscale image of the latter, represented in Fig. 1, is obtained by microtomography, in laboratory MATEIS (INSA Lyon, France). This bitmap image contains $1000 \times 1200 \times 1000$ voxels, and its resolution is such that the length of each voxel is $25 \mu \mathrm{m}$. At this scale, the material consists of three phases: air-voids, fine aggregates and the surrounding cement paste. Air-voids, which are almost black, are characterized by a low gray level and a near ellipsoidal shape. Fine aggregates have various shapes and sizes as well as various gray levels: most of aggregates, corresponding to limestone, are light (i.e. their gray level is higher than that of the cement paste), whereas others, made of sand-limes, are darker, with a gray-level slightly lower than that of the cement paste.

A three-phases microstructure of the material, where fine aggregates, cement paste (matrix) and voids have been segmented, is derived from the original grayscale image using filters and segmentation techniques.

Several steps are necessary to deduce an accurate microstructure from the grayscale image in order to be able to perform precise mechanical computations. Specifically, image acquisition limitation as well as material special features have to be taken into account. Indeed, the image, locally noised, is first filtered and thresholded (Sec. 2.3). While performing these first two steps, a long-range bias on the luminosity appears and needs to be removed (Sec. 2.4). Due to the resolution of the image, grains are artificially connected. To correctly disconnect them (Sec. 2.5), a multi-scale processing based on watershed algorithm is used. Finally, darker particles are not detected during the thresholding step and need to be segmented separately (Sec. 2.6).

\subsection{Noise filtering and segmentation by thresholding}

In this section, a filter and segmentation by automatic thresholding is applied to the image. The limitations of such simple method are discussed and alleviated in Secs. 2.4, 2.5 and 2.6.

Prior to segmentation, a standard Gaussian filter is used to remove local noise in the microtomography image, by means of a convolution by the 3D Gaussian kernel $f_{s}$ :

$$
f_{s}(\mathbf{x})=(2 \pi s)^{-1 / 2} \mathrm{e}^{-\frac{\|\mathbf{x}\|^{2}}{2 s^{2}}},
$$


where $s$ is the kernel standard deviation and $\|\mathbf{x}\|^{2}=x_{i}^{2}$. In practice, high values of $f$ are cut whenever $\max _{i} x_{i}>R=6 s, R$ being the size of the Gaussian filter. Two threshold values $\left(t_{1}, t_{2}\right)$ are then automatically derived from the field distribution to segment the image into three phases. More precisely, the following entropy maximization (see [20]) is applied to the field histogram. The image is partitioned into three classes defined as $C_{i}=$ $\left\{\mathbf{x} ; t_{i-1} \leq g(\mathbf{x})<t_{i}\right\}$, where $g(\mathbf{x})$ is the gray-level value at point $\mathbf{x}, i=1,2$ or $3, t_{0}=\min g(\mathbf{x})=0$ and $t_{3}=\max g(\mathbf{x})+1=256$. The probability density function of $C_{i}$, of volume $V_{i}$, is formally $p_{i}(g(\mathbf{x}) ; t)=\left(1 / V_{i}\right) \int_{C_{i}} \mathrm{~d}^{3} x \delta(t-g(\mathbf{x}))$ whereas its entropy is $H_{i}=H_{i}\left(t_{1}, t_{2}\right)=-\sum_{t_{i-1} \leq t<t_{i}} p_{i}(g(\mathbf{x}) ; t) \log p_{i}(g(\mathbf{x}) ; t)$. The total entropy $H\left(t_{1}, t_{2}\right)=\sum_{i=1,2,3} H_{i}\left(t_{1}, t_{2}\right)$ is maximized exactly by evaluating its values at each pair $\left(t_{1}, t_{2}\right)$. The values of $t_{1}$ and $t_{2}$ at a global maximum are used to segment the image in three classes $C_{1}, C_{2}$ and $C_{3}$.

The aggregates specific surface area measurement $S_{V}$, i.e. the aggregatesmatrix and aggregates-pores surface area divided by the image volume, is used as a means of optimizing the Gaussian filter size. As shown in Fig. 2, the specific surface area of a noisy image (left on the Fig.) is artificially increased. More precisely, when the filter size increases, the specific surface area $S_{V}$ decreases and stabilizes at $R \approx 5$, as shown in Fig. 3. Such value is used to perform the Gaussian filter and entropy maximization procedure, before further treatments are applied to the image.

\subsection{Removing luminosity long-range bias}

The previous segmentation procedure is unable to correct the non-uniform luminosity in the image at large-scales. Because of the cubic shape and the non negligible thickness of the sample, the luminosity of the image is indeed not uniform, due to the acquisition technique: along slices perpendicular to the rotation axis of the sample, the local average gray value of the image is larger on the center than it is on the edges. Along planes parallel to the rotation axis, the luminosity is much more uniform. As a result, only voids, whose contrast is very high, are correctly segmented, whereas aggregates are usually not (not shown).

To correct the luminosity bias, the original image is considered again, as well as the segmentation of pores obtained in the previous section. A local mean value, computed on a large area around each voxel, is subtracted from the grayscale image. More precisely, the value $g(\mathbf{x})$ at each voxel is replaced with $g(\mathbf{x})-\left\langle g^{\prime}\right\rangle_{2 D}(\mathbf{x}, d)$ where $\langle\cdot\rangle_{2 D}(\mathbf{x}, d)$ is a field average over a square of side length $2 d+1$, centered in $\mathbf{x}$ and perpendicular to the rotation axis, and 
$g^{\prime}(\mathbf{x}) \equiv g(\mathbf{x})$ in the aggregates and matrix whereas $g^{\prime}(\mathbf{x}) \equiv\langle g\rangle$ is constant in pores. The modified function $g^{\prime}$ has been introduced to minimize the effects of pores. Indeed, the porous phase is highly contrasted with the matrix and aggregates. In practice, out of several large values of $d=150,200,250,300$ pixels, it appears that the luminosity bias is best corrected when $d=250$.

The exact same procedure as given in section 2.3 is then applied to the image, after the luminosity bias has been removed. Overall, the resulting segmentation of fine aggregates is satisfactory. However three flaws need to be addressed: fine aggregates are connected to one another, which is not physically relevant (Sec. 2.5), dark fine aggregates are not segmented (Sec. 2.6), and the segmented aggregates contain artifacts such as holes of the size of a pixel, due to the remaining noise. This flaw is easily corrected by a standard holes filling algorithm. Indeed, holes are in this case voxels inside aggregates detected as matrix. In a binary image, the holes filling algorithm replaces all the background voxels not connected to the edge (hence inside the foreground) by foreground voxels. The foreground (resp. background) corresponds to fine aggregates (resp. matrix) in our case.

\subsection{Fine aggregates disconnection using watershed algorithm}

At this step, fine aggregates are much more connected in the segmented image than they are in reality. Indeed, the image resolution is not high enough to render thin matrix regions where aggregates are very close to one another, leaving many aggregates seemingly connected with their neighbors after the thresholding. Consequently, most of the particles appear artificially connected through a large percolating cluster. Such an effect is observed in Fig. 4 showing an image labeling where each label (i.e. grayscale value) corresponds to a cluster of connected particles. Ultimately, the effective properties of the resulting microstructure, if it was used as it is, would be much stiffer than that of the real material, assuming aggregates are stiffer than the matrix. For instance, in the case of quasi-rigid fine aggregates properties, the displacement of neighboring aggregates are usually not equal, as long as they remain separated. Since such information is not present in the original image, it must be included in the segmentation procedure. To this aim, a watershed algorithm is used to identify particles and disconnect them, as explained below.

The watershed algorithm ([4]) is a flooding process: water, starting from specified markers, "floods" the image, from the smallest to highest gray values. When two catchment basins meet, a dam is created, called "watershed 
plane". Specifically, the watershed algorithm applies well to the inverse of the distance function, computed from the binary image containing only interpenetrating inclusions (here, fine aggregates). The resulting watershed planes determine the aggregates frontiers with each other.

The separation of grains in a binary image consists thus in selecting markers, under the condition that there is a unique marker in each grain, and in using the watershed algorithm on the inverted distance function. To choose a marker in each grain, the local maxima of the distance function are commonly used. However if grains are not convex, the distance function has several maxima in a unique grain, which leads to an over-segmentation. To correct this, maxima are filtered. More precisely, maxima are cut by a size $h_{m}$, so only some of them, deeper than this prescribed threshold $h_{m}$, are kept as markers. If the value of $h_{m}$ is too high, some grains are not marked and the grains disconnection is not completely performed ; on the other hand, if this value is too low, some grains have several markers and there is an over-segmentation of the image.

It has been observed that $h_{m} \leq 2$ (markers in Fig. 5(a) for the case $\left.h_{m}=2\right)$ is necessary to achieve disconnection. However, this value implies an over-segmentation of the larger aggregates (Fig. 5(b)). Indeed, since the choice of the parameter $h_{m}$ is linked to the distance function of aggregates, hence to their size, the multi-scale nature of mortar should be taken into account. Accordingly, the method used to disconnect aggregates consists then in using, instead of previous markers, multi-scale markers (Fig. 5(c)) such as $h_{m}=2$ for smaller aggregates (of size less than 4), and $h_{m}=20$ for other aggregates. These last markers allow disconnecting aggregates from one another without segmenting larger aggregates, as shown in Fig. 5(d).

\subsection{Dark fine aggregates segmentation}

Although the thresholding method described above applies well to limestones, which appear as bright particles on the image, it cannot segment sand-limes, i.e. dark particles. Although the latter are only a tiny portion of all aggregates, they are lowly contrasted with the matrix, and a specific method is necessary to segment them. This method is described below, referring to Fig. 6, where a 2D section of the microstructure is given at each step of the procedure.

To that end, a holes filling algorithm is used, as explained in section 2.4 but extended to grayscale images. In the grayscale version, the algorithm "fills" basins not connected to the edge, hence making dark zones mostly 
uniform. Such technique is applied to the grayscale image after filtering, where the dark zones are made up of voids and sand-limes (Fig. 6(b)). Two methods are investigated: selection of "flat zones" with a volume higher than a prescribed threshold (Fig. 6(d)) and segmentation by thresholding (Fig. 6(e)). Each of these methods implies a parameter which is chosen to segment all darker fine aggregates. They are manually chosen as follows: flat zones with a volume higher than 20000 voxels are kept, whereas in the second method zones are thresholded between 2 and 133 (out of a maximum of 255). As it turns out, voids and dark aggregates are included in the flat zones as detected by both methods (Fig. 6(c)). However, the latter also include extra zones that should be removed: regions of the matrix surrounding voids as well as extra zones, located at different places in each method, that are neither voids nor dark aggregates. Accordingly, the dark aggregates are recovered by eliminating voids and taking the intersection of both results. To exclude voids, a mask is created by sufficiently dilating pores. The dilation size is chosen as a compromise: small enough to detect dark aggregates and sufficiently large to cover regions surrounding voids. A size of 3 voxels for the dilation is taken. Finally, after the intersection of the two results has been taken, small regions that are not dark aggregates subsist (Fig. 6(f)). A filter by "erosion-reconstruction" of size 2 voxels is applied to delete them. Equivalently, connected components of size less or equal to 2 are removed (Fig. 6(g)).

In the final segmented image, a 3-phase microstructure (Fig. 7) is obtained, where aggregates, matrix and voids represent 33.4\%, 64.0\% and $2.35 \%$, respectively, of the total volume. In turn, dark aggregates, i.e. sandlimes account for $0.785 \%$ of the microstructure volume. The volume fractions of aggregates and voids are lower than that of the real mortar material because they only take into account the largest ones, due to the resolution of the image. A comparison of the size distributions of aggregates used for the formulation of the mortar and measured by image analysis on the microtomography shows some discrepancy (Fig. 8): larger aggregates (with diameter larger than $3.15 \mathrm{~mm}$ ) were not present in the specimen, despite the fact that they should have a $4.2 \%$ volume fraction. This fact, reflecting the multi-scale nature of the mortar, is due to the relative size of these particles and of the sample image, and is considered further in the section about the RVE (Sec. $4)$. 


\section{Local and effective elastic response of mortar}

The local linear elastic response in the matrix and fine aggregates is defined by means of the local linear elastic tensor $\mathbb{L}$, which relates the secondorder strain and stress tensor fields $\varepsilon$ and $\sigma$ by:

$$
\sigma_{i j}=L_{i j, k l} \varepsilon_{k l}
$$

where the stress (resp. strain) field satisfies the equilibrium (resp. admissibility) condition, i.e.:

$$
\partial_{i} \sigma_{i j}=0, \quad \varepsilon_{k l}=(1 / 2)\left(\partial_{k} u_{l}+\partial_{l} u_{k}\right) .
$$

In the above, the local displacement field is noted $u_{k}$, and small deformation is assumed.

In the classical framework of the homogenization of composite media, assuming perfect interfaces between fine aggregates and cement paste, the effective elastic response is defined through the averages:

$$
\widetilde{\sigma}_{i j}=\widetilde{L}_{i j, k l} \widetilde{\varepsilon}_{k l}, \quad \widetilde{\sigma}=\langle\sigma\rangle, \quad \widetilde{\varepsilon}=\langle\varepsilon\rangle,
$$

where $\widetilde{\mathbb{L}}$ is the macroscopic elastic tensor, relating the material overall stress $\widetilde{\sigma}$ and strain $\widetilde{\varepsilon}$, and $\langle\cdot\rangle$ denotes an average over the whole volume. Assuming isotropy of the local elastic tensor $\mathbb{L}$, and of the microstructure geometry, the effective elastic tensor $\widetilde{\mathbb{L}}$ is isotropic. Both are then decomposed as:

$$
\begin{aligned}
L_{i j, k l}(\mathbf{x}) & =\mu(\mathbf{x})\left(\delta_{i k} \delta_{j l}+\delta_{i l} \delta_{j k}\right)+[\kappa(\mathbf{x})-(2 / 3) \mu(\mathbf{x})] \delta_{i j} \delta_{k l}, \\
\widetilde{L}_{i j, k l} & =\widetilde{\mu}\left(\delta_{i k} \delta_{j l}+\delta_{i l} \delta_{j k}\right)+[\widetilde{\kappa}-(2 / 3) \widetilde{\mu}] \delta_{i j} \delta_{k l},
\end{aligned}
$$

where $\delta$ is the Kronecker symbol, $\kappa$ (resp. $\mu$ ) are the phase-dependent local bulk (resp. shear) modulus, and $\widetilde{\kappa}$, (resp. $\widetilde{\mu}$ ) their macroscopic counterparts. In voids, $\mu(\mathbf{x}) \equiv \kappa(\mathbf{x}) \equiv 0$, i.e. stress is zero and strain is undetermined, whereas $\mu(\mathbf{x}) \equiv \mu^{(a)}, \kappa(\mathbf{x}) \equiv \kappa^{(a)}$ in aggregates and $\mu(\mathbf{x}) \equiv \mu^{(m)}, \kappa(\mathbf{x}) \equiv \kappa^{(m)}$ in the matrix or cement paste. In fine aggregates and in the matrix, the local elastic moduli are equivalently determined by the Poisson ratio and the Young modulus, resp.:

$$
\nu(\mathbf{x})=\frac{1}{2} \frac{3 \kappa(\mathbf{x})-2 \mu(\mathbf{x})}{3 \kappa(\mathbf{x})+\mu(\mathbf{x})}, \quad E(\mathbf{x})=\frac{9 \kappa(\mathbf{x}) \mu(\mathbf{x})}{3 \kappa(\mathbf{x})+\mu(\mathbf{x})} .
$$




\begin{tabular}{|c|c|c|}
\hline Time after loading $(t)$ & $E^{(a)} / E_{\text {fictive }}^{(m)} \min$ & $E^{(a)} / E_{\text {fictive }}^{(m)} \max$ \\
\hline \hline 1 min & 3.73 & 3.73 \\
\hline 1 day & 3.75 & 4.11 \\
\hline 1 year & 7.29 & 13.0 \\
\hline 10 years & 11.2 & 40.8 \\
\hline 100 years & 25.5 & 317 \\
\hline
\end{tabular}

Table 1: Minimum and maximum contrasts encountered in the underlying $M$ elastic homogenization computations required to estimate the effective creep function at time $t$.

Consistently with values used by Wriggers et al [24] and with values measured by Granger [6], the Poisson ratio is taken as a phase-independent constant equal to 0.2. As a result, the problem only depends on the Young modulus aggregate-matrix contrast, defined as $\chi \equiv E^{(a)} / E^{(m)}$. Choice has been made to explore values of contrast $\chi$ equal to $10^{-8}, 3,100,1000$ and $10^{4}$. The lowcontrast value of 3 corresponds to the true Young's modulus ratio $([24,6])$. Values smaller (resp. greater) than 3 let us anticipate needs for damage (resp. creep) computations. Indeed, by combining the correspondence principle between linear visco-elasticity in the real domain and linear elasticity in the Laplace-Carson domain with a numerical inversion procedure, the effective creep function is evaluated by considering $M$ elastic homogenization problems at each time $t$ (with typically $M=20$ ). In the case of the homogenization of concrete, considering a viscoelastic matrix and elastic aggregates, Sanahuja et al [18] estimated $M$ fictive Young moduli for the matrix phases at various time $t$ (see Tab. 1).

\subsection{Boundary and loading conditions}

The overall and local fields response are studied by applying two types of loading conditions. The material is subjected to hydrostatic strain loading as well as shear strain loading defined respectively as:

$$
\left\langle\varepsilon_{i j}\right\rangle=\varepsilon_{0} \delta_{i j}, \quad\left\langle\varepsilon_{i j}\right\rangle=\left\{\begin{array}{l}
\varepsilon_{0} \text { if } i j=x y \text { or } i j=y x, \\
0 \text { otherwise }
\end{array}\right.
$$

where $\varepsilon_{0}$ is a scalar. In turn, the boundary conditions considered in this work consist of one of the average field condition above and of periodic boundary conditions, i.e. the traction vector $\sigma \cdot \mathbf{n}$ at the boundary of normal $\mathbf{n}$ is antiperiodic, and the strain field $\varepsilon$ is periodic. Although the microstructure is 
not in itself periodic, such conditions are known to minimize edge effects (see [12]). It is noted that the local field response, in particular the strain and stress fields histograms investigated in this work, depends on the loading.

\subsection{Fast Fourier transform method}

Full-field computations are undertaken using the "augmented Lagrangian" algorithm [14], one of the Fast Fourier Transform methods originally introduced in [15] that is especially efficient for handling infinitely-contrasted media. One of the main advantage of such method is its capability to handle large-size systems $[22,23]$. Such algorithms make use of the LippmannSchwinger equations (Eq. 7), i.e. the local material properties are computed in the real space whereas strain admissibility and stress equilibrium conditions (Eq. 3) are enforced in the Fourier domain:

$$
\begin{aligned}
\varepsilon_{i j}(\mathbf{x}) & =\left\langle\varepsilon_{i j}\right\rangle+\int \mathrm{d}^{3} \mathbf{x}^{\prime} G_{i j k l}^{(0)}\left(\mathbf{x}-\mathbf{x}^{\prime}\right) \tau_{k l}\left(\mathbf{x}^{\prime}\right), \\
\tau_{i j}(\mathbf{x}) & \equiv \sigma_{i j}(\mathbf{x})-L_{i j k l}^{(0)}: \varepsilon_{k l}(\mathbf{x}),
\end{aligned}
$$

where $\mathbb{L}^{(0)}$ is a given "reference" elastic tensor and $\mathbb{G}^{(0)}$ is its associated Green's function. Eqs. (7a) and (7b) are computed in the Fourier domain and real space resp., with Fast Fourier transforms used to iterate between the two. The authors refer to [14] for the details of the augmented Lagrangian scheme, not recalled here. It should be noted that an alternative "stress formulation" of the "augmented Lagrangian" method, that uses the stress, instead of the strain Green's function, exist. Convergence of the latter proves to be more efficient when the local elastic moduli of inclusions are very high compared to that of the matrix. However, in the presence of voids, no such improvement has been observed, thus the standard strain formulation was used in the present case.

In this work, full-field computations are directly applied to cubic grids of length either 500 or 735 voxels (i.e. $1.25 \mathrm{~cm}$ and $1.84 \mathrm{~cm}$ resp.). The latter discretization was used at low and high contrast $\chi=E^{(a)} / E^{(m)}=3$ and $\chi=10^{4}$, resp. The advantage of the method using the FFT is that we do not need to mesh the complex microstructure, and that we obtain the field at the same scale as the microstructure.

\subsection{Effective behavior}

The effective bulk (resp. shear) modulus $\widetilde{\kappa}$ (resp. $\widetilde{\mu}$ ) is computed numerically for various values of the aggregates-matrix contrast $\chi$, with hydrostatic 


\begin{tabular}{|c||c|c|c|c|c|}
\hline$\chi=E^{(a)} / E^{(m)}$ & $10^{-8}$ & 3 & 100 & 1000 & $10^{4}$ \\
\hline \hline$\widetilde{\kappa} / \kappa^{(m)}$ & 0.331 & 1.384 & 2.72 & 3.49 & 4.66 \\
\hline$\kappa_{H S^{+}} / \kappa^{(m)}$ & 0.463 & 1.490 & 21.897 & 211.3 & 2096.93 \\
\hline$\kappa_{\mathrm{SC}} / \kappa^{(m)}$ & 0 & 1.373 & 2.680 & 2.851 & 2.871 \\
\hline \hline$\widetilde{\mu} / \mu^{(m)}$ & 0.34 & 1.385 & 2.858 & 3.785 & 6.245 \\
\hline$\mu_{H S^{+}} / \mu^{(m)}$ & 0.461 & 1.490 & 21.890 & 211.2 & 2096.93 \\
\hline$\mu_{\mathrm{SC}} / \mu^{(m)}$ & 0.286 & 1.373 & 2.681 & 2.851 & 2.870 \\
\hline
\end{tabular}

Table 2: Effective bulk and shear moduli ( $\widetilde{\kappa}$ and $\widetilde{\mu}$ resp.) at various aggregates/matrix contrasts $\chi$ (first row): numerical FFT computations (second and fifth row), upper HashinShtrikman's bounds ( $\mathrm{HS}^{+}$, rows 3 and 6 ), and the self-Consistent estimate (SC, row 4 and 7). Column 2 corresponds to the lowest contrast (i.e. aggregates behave as pores) whereas column 6 on the right corresponds to quasi-rigid aggregates.

(resp. shear) strain loading. More precisely, the local bulk and shear moduli are fixed in the matrix, whereas the elastic response of fine aggregates varies with the contrast. The FFT results are compared with Hashin-Shtrikman's upper bound $\left(\mathrm{HS}^{+}\right)[10]$ and a self-consistent (SC) estimate. The latter is given by the reference elastic tensor that is self-consistent with HashinShtrikman's variational principle. Results are given in Tab. 2. Contrary to Hashin and Shtrikman's upper bound, the effective bulk and shear moduli as computed by FFT tend to finite values at large contrasts. In effect, in Hashin's coated spheres model for a two-phase material, the embedding phase percolates and the embedded phase does not. Accordingly, Hashin and Shtrikman's upper (resp. lower) bounds are relevant when the stiffest (resp. weakest) phase percolates. FFT results are accordingly far apart from HS bounds, as the aggregates do not percolate. Hashin and Shtrikman's lower bounds however are zero here due to the presence of the porous phase, albeit of small volume fraction. In contrast, the self-consistent method provides estimates much closer to the FFT results at low contrast. It however significantly differs when fine aggregates are quasi-rigid (i.e. $\chi \geq 100$ ).

An effective Young modulus of value 26.4 GPa has been experimentally measured on a sample mortar comparable to the one which is considered in this study. The homogenization by numerical FFT computation in the case $E^{(m)}=20 \mathrm{MPa}, E^{(a)}=60 \mathrm{MPa}, \nu^{(m)}=\nu^{(a)}=0.2$ gives an effective Young modulus value of $\widetilde{E}=27.7 \mathrm{GPa}$, which is consistent with the experimental value. 


\section{Statistical characterization of the macroscopic response: Rep- resentative Volume Element (RVE)}

\subsection{Evaluation of the Representative Volume Element}

The Representative Volume Element (Kanit et al, 2003, [12]) or RVE is evaluated using full-fields computations undertaken with the FFT method. It is defined here as the size of the volume giving the effective property with a given precision $\epsilon$. Its evaluation is based on the following property: considering a random variable $Z$ defined on a total volume of size $V_{0}$ :

$$
\frac{D_{Z}^{2}(V)}{D_{Z}^{2}} \sim A_{3}\left(\frac{1}{V}-\frac{1}{V_{0}}\right)
$$

when sub-volumes $V$ of $V_{0}$ are such as $V \gg A_{3}$, with $A_{3}$ the integral range. $D_{Z}^{2}$ is the point variance over the total volume $V_{0}$ and $D_{Z}^{2}(V)$ is the variance of the spatial means of $Z$ over disjointed sub-volumes $V$. In practice, the volume $A_{3}$ is evaluated by a fit of Eq. 8 . The RVE size is then deduced by:

$$
V_{R V E}=\frac{4 D_{Z}^{2} A_{3}}{\epsilon^{2}\langle Z\rangle^{2}}
$$

with $\langle Z\rangle$ the mean of $Z$ over $V_{0}$. Conversely, Eq. (9) is also used to evaluate the precision $\epsilon$ of an effective property determined from a volume $V_{0}$. When shear loading is applied, $Z(\mathbf{x})=\sigma_{x y}(\mathbf{x})$ ( $x y$ being the macroscopic shear direction) is chosen, whereas for hydrostatic strain loading, $Z(\mathbf{x})=\sigma_{m}(\mathbf{x})$ is chosen, where $\sigma_{m}=(1 / 3) \sigma_{i i}$ is the mean stress field. The "morphological RVE" is evaluated separately for each phase, and equal to the RVE of its characteristic function. More precisely, $\langle Z\rangle=p_{i}$ and $D_{Z}^{2}=p_{i}\left(1-p_{i}\right)$ are the volume fraction and point variance, resp., of phase $i$, whereas the term $D_{Z}^{2}(V)$ is computed numerically.

\subsection{Mechanical and morphological RVE}

The Representative Volume Element (RVE) is evaluated for both the bulk and shear moduli $\widetilde{\kappa}$ and $\widetilde{\mu}$, at various aggregates-matrix contrasts $\chi$. A fixed precision $\epsilon=5 \%$ is prescribed (Tab. 3). It appears that a precision of $\epsilon=5 \%$ is achieved at low contrast $\chi=3$ or $\chi \leq 100$ and at $\chi=10^{-8}$ for volume of size larger than $V_{0}=500^{3}$ voxels. In the rigid case, i.e. at higher contrasts $\chi=1000,10^{4}$, the precision of the results is evaluated, for $V_{0}=500^{3}, 735^{3}$ respectively in Tab. (4). Finally, the morphological RVE is 


\begin{tabular}{|c||c|c|}
\hline$\chi=E^{(a)} / E^{(m)}$ & $V_{R V E}^{\kappa}$ & $V_{R V E}^{\mu}$ \\
\hline \hline $10^{-8}$ & $1.1^{3} \mathrm{~cm}^{3}\left(433^{3}\right.$ voxels $)$ & $1.3^{3} \mathrm{~cm}^{3}\left(515^{3}\right.$ voxels $)$ \\
\hline 3 & $0.62^{3} \mathrm{~cm}^{3}\left(249^{3}\right.$ voxels $)$ & $0.62^{3} \mathrm{~cm}^{3}\left(248^{3}\right.$ voxels $)$ \\
\hline 100 & $1.0^{3} \mathrm{~cm}^{3}\left(414^{3}\right.$ voxels $)$ & $1.1^{3} \mathrm{~cm}^{3}\left(428^{3}\right.$ voxels $)$ \\
\hline 1000 & $1.4^{3} \mathrm{~cm}^{3}\left(574^{3}\right.$ voxels $)$ & $1.3^{3} \mathrm{~cm}^{3}\left(528^{3}\right.$ voxels $)$ \\
\hline $10^{4}$ & $3.2^{3} \mathrm{~cm}^{3}\left(1270^{3}\right.$ voxels $)$ & $2.6^{3} \mathrm{~cm}^{3}\left(1037^{3}\right.$ voxels $)$ \\
\hline
\end{tabular}

Table 3: Size of the RVE at the prescribed precision $\epsilon=5 \%$ for the effective bulk and shear moduli, $\widetilde{\kappa}$ and $\widetilde{\mu}$ resp., for various contrasts $\chi$.

\begin{tabular}{|c||c|c|}
\hline$\chi=E^{(a)} / E^{(m)}, V_{0}$ & $\epsilon_{\kappa}$ & $\epsilon_{\mu}$ \\
\hline \hline$\chi=1000, V_{0}=500^{3}$ & $6.2 \%$ & $5.4 \%$ \\
\hline$\chi=10^{4}, V_{0}=735^{3}$ & $11.4 \%$ & $8.4 \%$ \\
\hline
\end{tabular}

Table 4: Relative precision $\epsilon$ obtained on the bulk and shear moduli $\widetilde{\kappa}$ and $\widetilde{\mu}$ resp., for a volume $V_{0}=500^{3}$ and $735^{3}$ voxels, at contrast $\chi=1000$ and $10^{4}$ resp.

evaluated for $\epsilon=5 \%$ in Tab. 5. The resulting large RVE size obtained for voids is a consequence of its low volume fraction. Overall, it appears that $V_{\mathrm{RVE}}^{\text {elastic fields }} \simeq V_{\mathrm{RVE}}^{\text {aggregate }}$ : aggregates control elastic fields variability, and then the RVE size.

\subsection{Corrected RVE}

As noticed earlier in this paper, the largest aggregates (with diameter larger than $3.15 \mathrm{~mm}$ and $4.2 \%$ volume fraction) are missing in the image. The size of the RVE corresponding to these aggregates (for the volume fraction, and correspondingly for the mechanical fields as seen above) can be estimated with the integral range technique for a random location of aggregates. For a $5 \%$ relative precision, a volume of size $6.7^{3} \mathrm{~cm}^{3}$ should be used, which is a little bit more than two times larger than the RVE for homogenization of elastic properties $\left(2.6^{3} \mathrm{~cm}^{3}\right)$, and consistent with the size of specimens used to measure the effective properties. For further studies, a multi-scale random model of mortar could help us to simulate RVE's of this size. With

\begin{tabular}{|c|c|c|}
\hline$V_{R V E}^{\text {voids }}$ & $V_{R V E}^{\text {matrix }}$ & $V_{R V E}^{\text {aggregates }}$ \\
\hline $4.2^{3} \mathrm{~cm}^{3}\left(1676^{3}\right.$ voxels $)$ & $1.0^{3} \mathrm{~cm}^{3}\left(407^{3}\right.$ voxels $)$ & $1.5^{3} \mathrm{~cm}^{3}\left(619^{3}\right.$ voxels $)$ \\
\hline
\end{tabular}

Table 5: Morphological RVE for each phase, for $\epsilon=5 \%$ 


\begin{tabular}{|c||c|c|c|c|c|}
\hline Contrast $\chi=E^{(a)} / E^{(m)}$ & $10^{-8}$ & 3 & 100 & 1000 & 10000 \\
\hline \hline$\alpha$ (hydrostatic strain loading) & -8 & -17 & -7.6 & -7 & -7.5 \\
\hline$\alpha$ (shear strain loading) & -7.6 & -13.6 & -6 & -5.4 & -5.2 \\
\hline
\end{tabular}

Table 6: Distribution tail of the parallel stress field fitted with a power law $P_{\sigma_{m}}(t) \simeq t^{\alpha}$ for $t \gg 1$

the present size used for calculations, the corrected relative precision of the volume fraction of granulates can be estimated to $20 \%$, in order to account for the missing largest granulates of the size distribution.

\section{Field maps and field histograms}

2D sections of fields maps of the mean stress field $\sigma_{m}$ (opposite of pressure) and of the shear stress field $\sigma_{x y}$ are shown in Fig. 9 and 10 resp., for hydrostatic and shear stress loading. Note that both field components are parallel to the applied loading in the sense that their average is non-zero. When contrast is high, i.e. grains are quasi-rigid, a concentration of strain is found in-between aggregates when hydrostatic loading is applied. Localization patterns are even stronger when the material is subjected to shear.

Histograms of $\sigma_{m}$ and $\sigma_{x y}$, at applied hydrostatic and shear strain loading resp., are given in Fig. 11, at various contrasts. At the lowest contrast $\chi=3$, the field histogram $P_{\sigma_{m}}(t)$ of the mean stress field is a sharp, nearlysymmetrical distribution close to a Gaussian, except at very high values of $t=\sigma_{m}(\mathbf{x})$. As such, the variance of the field increases with the contrast, and a lack of symmetry of the distribution appears.

For each histogram, the distribution tail is fitted with a power law $P_{\sigma_{m}}(t) \sim$ $t^{\alpha}$ for $t \gg 1$ where $\alpha<0$. The values of $\alpha$ are given in Tab. 6 for various contrasts $\chi$ and loadings. For the shear strain loading case, for $\chi \geq 3, \alpha$ increases when the contrast $\chi$ increases, i.e. zones of high mean stress in the matrix are more likely to occur when the contrast is high or equivalently when aggregates are quasi-rigid. Overall, it is proved that $\alpha \simeq-7.5$ at infinite contrast $\chi=0$ or $\chi=+\infty$, except when $\chi=+\infty$ and shear loading is applied, where $\alpha \simeq-5.2$. 


\section{Local stress field response}

\subsection{Morphological tools for measuring spatial set correlations}

In this section, the localization of regions of the matrix subjected to high elastic stress, with respect to fine aggregates or voids, is studied in detail. To this aim, the correlation between one of the material phases (i.e. aggregates or voids) and a component of the stress field is quantitatively characterized by means of morphological tools presented below [11]. Such tools are based on the use of the dilation operation [19] of a set $A$ by a structuring element (of increasing size $r$ ) $B(r)$ defined as $A \oplus B(r)=\left\{\cup B_{\mathbf{x}}(r), \mathbf{x} \in A\right\}$, with $B_{\mathbf{x}}(r)$ the translation of $B(r)$ by $\mathbf{x}$. The structuring element $B(r)$ used for dilations is a rhombicuboctahedron of size $r$, to numerically approximate a ball.

First, the mean $m_{j}(r)$ of the stress field in the matrix is measured as a function of the distance $r$ to a given set $A_{j}$ by averaging the field on the region $\left.\left.X_{r}=\left(A_{j} \oplus B(r)\right)\right) \cap\left(A_{j} \oplus B(r-1)\right)\right)^{c} \cap A_{m}$ (Fig. 12), where $\cdot{ }^{c}$ denotes the complementary set of $\cdot$. In the following, the set $A_{m}$ is the matrix phase, and the set $A_{j}$ is either the fine aggregates phase or the skeleton by influence zone (SKIZ) [13] of the aggregates, noted $m_{a}(r)$ and $m_{s}(r)$. The latter is determined in 3D by taking the watershed of the distance function to the aggregates (Fig. 13). Contrary to the former, regions far from the aggregates correspond to small values of $r$.

A second morphological tool, based on the increasing neighborhoods method, is used to measure the spatial "arrangement" between two sets. This method evaluates, for increasing values of $r$, the function $\rho_{j}$ which is defined as

$$
\rho_{j}(r)=\frac{\Phi_{j}(r)}{P\left\{x \in\left(A_{k} \oplus B(r)\right)\right\}-p_{k}} \frac{1-p_{k}}{p_{j}},
$$

where $p_{j}$ (resp. $p_{k}$ ) is the volume fraction of the phase $A_{j}$ (resp. $A_{k}$ ) and $\Phi_{j}(r)=P\left\{x \in\left(A_{k} \oplus B(r)\right) \cap A_{j}\right\}$ (Fig. 14). This definition is equivalent to $\rho_{j}(r)=\frac{F_{j}(r)}{F(r)}$, with $F(r)=P\left\{d\left(x, A_{k}\right)<r \quad \mid \quad x \in A_{k}^{c}\right\}=\frac{P\left\{x \in\left(A_{k} \oplus B(r)\right)\right\}-p_{k}}{1-p_{k}}$ the distribution of distances of a random point $x$ in $A_{k}^{c}$ to the boundary of $A_{k}$, and $F_{j}(r)=P\left\{d\left(x, A_{k}\right)<r \quad \mid \quad x \in A_{j}\right\}=\frac{\Phi_{j}(r)}{p_{j}}$ the distribution of distances of a random point $x$ in $A_{j}$ to the boundary of $A_{k}$. Values of $\rho_{j}(r)$ are then interpreted at the scale $r$ as a preferential association (resp. a repulsion effect) between sets $A_{k}$ and $A_{j}$ when $\rho_{j}(r)>1$ (resp. $\rho_{j}(r)<1$ ). Hereafter, the increasing neighborhoods method is applied to one of the material phases 
and to a set obtained by thresholding one of the stress field component in the matrix (resp. $A_{j}$ and $A_{k}$ in the equations above).

The component of the stress field parallel to the applied loading is considered hereafter, i.e. the mean (resp. shear) stress component $\sigma_{m}$ (resp. $\sigma_{x y}$ ), when hydrostatic (resp. shear) strain loading is applied.

\subsection{Results}

Graphs of the functions $m_{a}(r)$ and $m_{s}(r)$ are shown in Fig. 15 for various contrasts and with either hydrostatic or shear strain loading. At high contrasts, it appears that the parallel stress field highest values are located at large distance $r$ of the aggregates. Indeed, at $\chi=10^{4}$ and $\chi=10^{3}$, independently of the loading direction, $m_{a}(r)$ increases with $r$ (Figs. 15(a) and 15(b), except at a few points), whereas the inverse trend is observed for $m_{s}(r)$ (Figs. 15(c) and 15(d)), i.e. when the distance is measured from the aggregates SKIZ. Equivalently, the region of the matrix near the aggregates SKIZ is subjected to strain and stress fields that are higher than the average applied field. In particular, such regions are candidate for developing damage zones. Such observation is made as well in Fig. 16, where a map of the stress field is shown, with the aggregates SKIZ superimposed in blue.

The function $\rho_{j}(r)$ is evaluated by taking the aggregates phase or the voids as the set $A_{j}$, and noted $\rho_{a}(r)$ and $\rho_{v}(r)$, resp. In each case, $A_{k}$ is the region of the matrix where the parallel stress component is higher than a chosen threshold. This threshold is adjusted so that the volume of $A_{k}$ is $5 \%$ of the domain. Accordingly, low values of $r$ correspond to regions subjected to high stress. A 3D map of such thresholded stress field is shown in purple and green in Fig. 17.

The resulting functions $\rho_{a}(r)$ and $\rho_{v}(r)$ are plotted as functions of the distance $r$ at various contrasts $\chi$, with hydrostatic and shear strain loading conditions in Fig. 18. Consistently with the results obtained using the mean stress functions $m_{a, s}(r)$, regions of high stress are not located close to grains. The grains concentration is indeed significantly lower than 1 when $r$ is small, i.e. in regions subjected to high stress, independently of the loading direction (Figs. 18(a) and 18(b)). Additionally, at high contrasts, high stress regions are not located around voids. Indeed, as shown in Figs. 18(c) and 18(d), the function $\rho_{v}(r)$ is always smaller than 1 , except at low-contrast and when the grains behave as voids ( $\chi=3$ and $\chi=10^{-8}$, resp.). At low aggregates $/$ matrix contrast, indeed, the effect of aggregates is negligible and the elastic response 
is analogous to that of a matrix with isolated voids, around which stress is concentrated.

\section{Conclusion}

In this study, the linear elastic properties of a sample of mortar composite has been investigated, using a combined FFT/morphological approach. A microtomography image is segmented using morphological tools. Numerical homogenization is handled by a FFT-based tool that is directly applied to the microstructure image. Compared to the classical self-consistent model, full-fields numerical homogenization is necessary to render accurately the effective elastic response of mortar at high contrast of properties between the matrix and fine aggregates. The representative volume element (RVE) was evaluated at various contrasts. It is found to be in the order of magnitude of the aggregate phase RVE, confirming that aggregates phase controls fields variability.

Moreover, a morphological analysis of the elastic response in the matrix indicates that, for level of contrasts ratio such as $\chi \geq 10^{3}$, regions subjected to high stress are located preferentially far from fine aggregates. As a first approximation, a candidate for such regions is the SKIZ of the fine aggregates phase, whether hydrostatic or shear strain loading is applied. The use of the SKIZ to study the stress localization could be improved by taking into account either the privileged loading directions, or the aggregates size. This would lead to a more accurate determination of the high-stress regions. That analysis highlights the way the concentration of stress is localized in some particular zones of the cement paste. It is a step to the understanding of creep localization.

The methods presented in this paper are general and could be extended to study the elastic response of random simulations of concrete microstructures, allowing a refined characterization of the impact of morphological parameters on effective properties, and on the stress localization in the material.

\section{References}

[1] B. Bary, M.B. Haha, E. Adam, P. Montarnal, Numerical and analytical effective elastic properties of degraded cement pastes, Cement and Concrete Research 39 (2009) 902-912. 
[2] D. Bentz, Three-dimensional computer simulation of portland cement hydration and microstructure development, Journal of the American Ceramic Society 80 (1997) 3-21.

[3] F. Bernard, S. Kamali-Bernard, W. Prince, 3d multi-scale modelling of mechanical behaviour of sound and leached mortar, Cement and Concrete Research 38 (2008) 449-458.

[4] S. Beucher, F. Meyer, Mathematical morphology in image processing, Mathematical Morphology in Image Processing, 1992, pp. 433-481.

[5] A. Caballero, C. López, I. Caroll, 3d meso-structural analysis of concrete specimens under uniaxial tension, Computer Methods in Applied Mechanics and Engineering 195 (2006) 7182-7195.

[6] L. Granger, Comportement différé du béton dans les enceintes de confinement de centrales nucléaires, analyse et modélisation d'ouvrage, Ph.D. thesis, Laboratoire Central des Ponts et Chaussées, 1996.

[7] C.J. Haecker, E. Garboczi, J. Bullard, R. Bohn, Z. Sun, S. Shah, T. Voigt, Modeling the linear elastic properties of portland cement paste, Cement and Concrete Research 35 (2005) 1948-1960.

[8] S. Häfner, S. Eckardt, T. Luther, C. Könke, Mesoscale modeling of concrete: Geometry and numerics, Computers and Structures 84 (2006) 450-461.

[9] M. Hain, P. Wriggers, Numerical homogenization of hardened cement paste, Computational Mechanic (2007) 197-212.

[10] Z. Hashin, S. Shtrikman, A variational approach to the theory of the elastic behaviour of multiphase materials, Journal of the Mechanics and Physics of Solids 11 (1963) 127-140.

[11] D. Jeulin, Study of spatial distributions in multicomponent structures by image analysis, Proceedings of the 4th European Symposium of Stereology, Acta Stereol 5/2 (1986) 233-239.

[12] T. Kanit, S. Forest, I. Galliet, V. Mounoury, D. Jeulin, Determination of the size of the representative volume element for random composites: statistical and numerical approach, International Journal of Solids and Structures 40 (2003) 3647-3679. 
[13] C. Lantuejoul, Skeletonization in quantitative metallography, Issues of Digital Image Processing 34 (1980) 107-135.

[14] J.C. Michel, H. Moulinec, P. Suquet, A computational scheme for linear and non-linear composites with arbitrary phase contrast, International Journal for Numerical Methods in Engineering 52 (2001) 139-160.

[15] H. Moulinec, P. Suquet, A fast numerical method for computing the linear and nonlinear mechanical properties of composites, Comptes rendus de l'Académie des sciences. Série II 318 (1994) 1417-1423.

[16] G. Nagai, T. Yamada, A. Wada, Stress analysis of concrete material based on geometrically accurate finite element modeling, Third International Conference on Fracture Mechanics of Concrete and Concrete Structures (FRAMCOS-3) 42 (1998) 1077-1086.

[17] A. Rollett, R. Lebenshon, M. Groeber, Y. Choi, J. Li, G.S. Roher, Stress hot spots in viscoplastic deformation of polycristals, Modelling and Simulation in Materials Science and Engineering 18 (2010).

[18] J. Sanahuja, C. Toulemonde, Numerical homogenization of concrete microstructures without explicit meshes, Cement and Concrete Research (submitted in 2010).

[19] J. Serra, Image analysis and mathematical morphology, Image Analysis and Mathematical Morphology, 1982.

[20] M. Sezgin, B. Sankur, Survey over image thresholding techniques and quantitative performance evaluation, Journal of Electronic Imaging 13 (2004) 146-165.

[21] V. Šmilauer, Z. Bažant, Identification of viscoelastic c-s-h behavior in mature cement paste by fft-based homogenization method, Cement and Concrete Research 40 (2010) 197-207.

[22] F. Willot, D. Jeulin, Elastic behavior of composites containing boolean random sets of inhomogeneities, International Journal of Engineering Science 47 (2009) 313-324.

[23] F. Willot, D. Jeulin, Elastic and electrical behavior of some random multiscale highly-contrasted composites, Journal of Multiscale Computational Engineering (to be published). 
[24] P. Wriggers, S. Moftah, Mesoscale models for concrete: Homogenisation and damage behaviour, Finite Elements in Analysis and Design 42 (2006) 623-636.

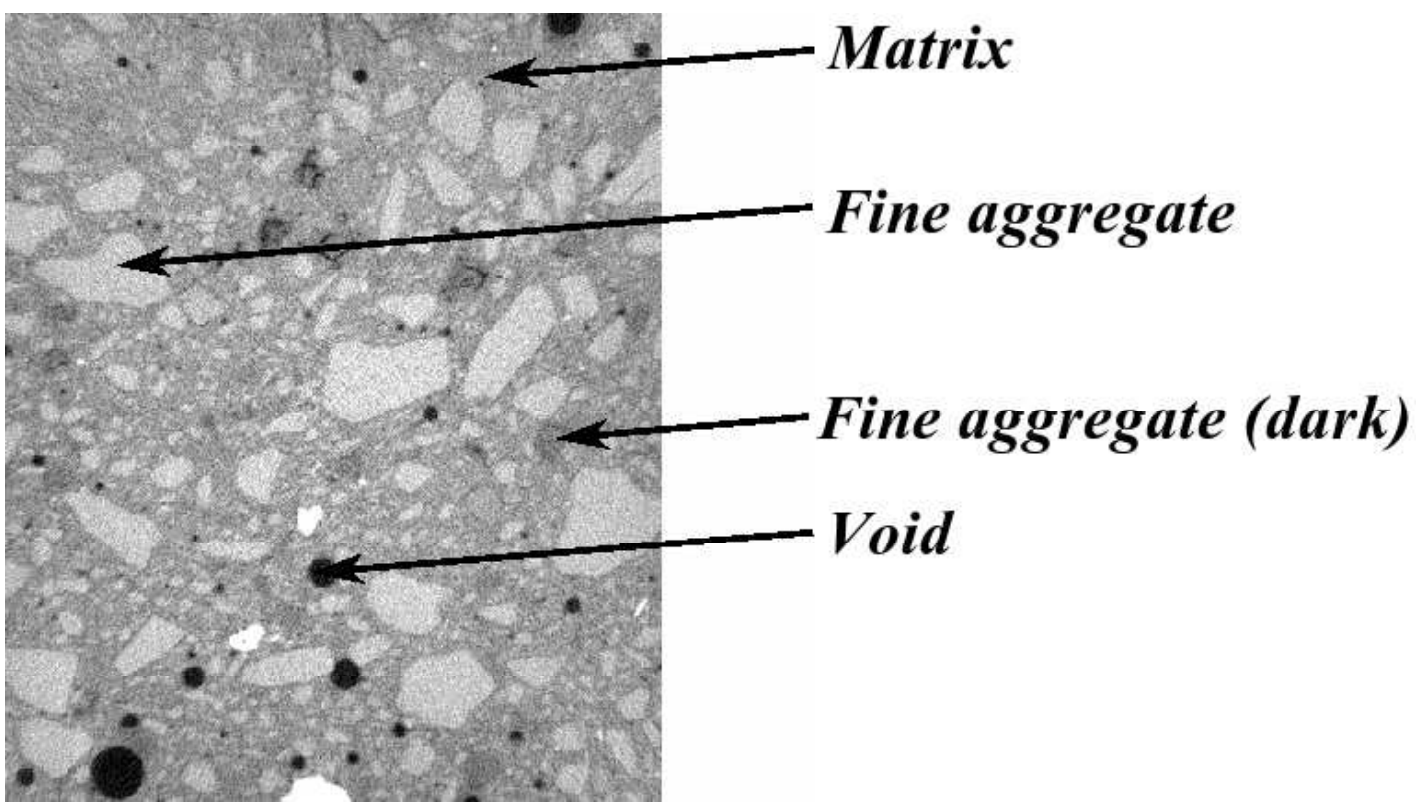

Figure 1: 2D section of a 3D mortar image obtained by microtomography: matrix/cement, light and dark fine aggregates, and voids (strongly dark). The 3D image is of size $2.5 \times 3 \times 2.5 \mathrm{~cm}^{3}$ and of resolution $25 \mu \mathrm{m} /$ voxel. 


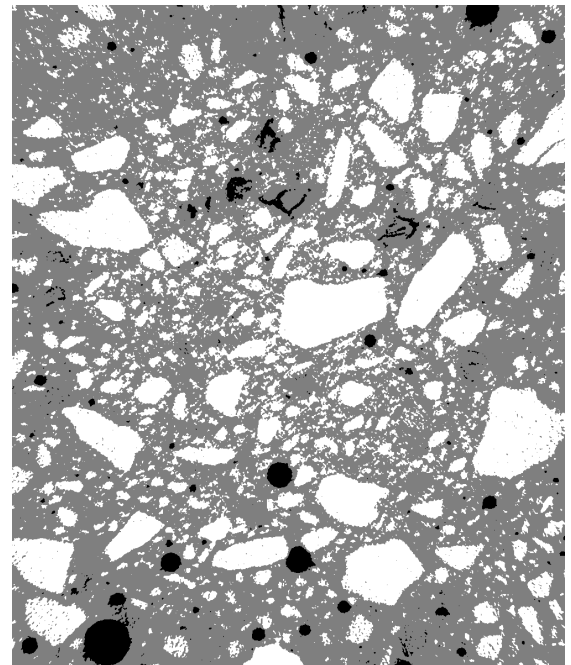

(a) $R=2$

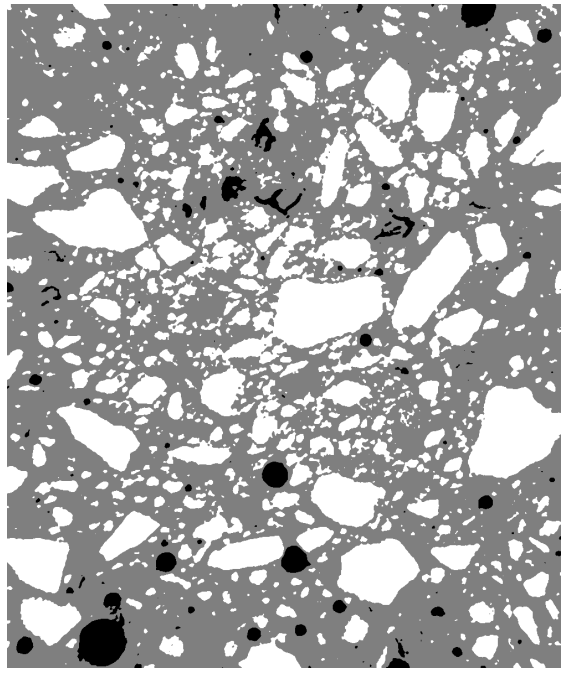

(b) $R=5$

Figure 2: Examples of filtered and thresholded images at two filter sizes $R=2,5$ voxels: fine aggregates (white), voids (black) and matrix (gray). When the image noise is incompletely removed ( $R=2$, left), the aggregates specific surface area is overestimated.

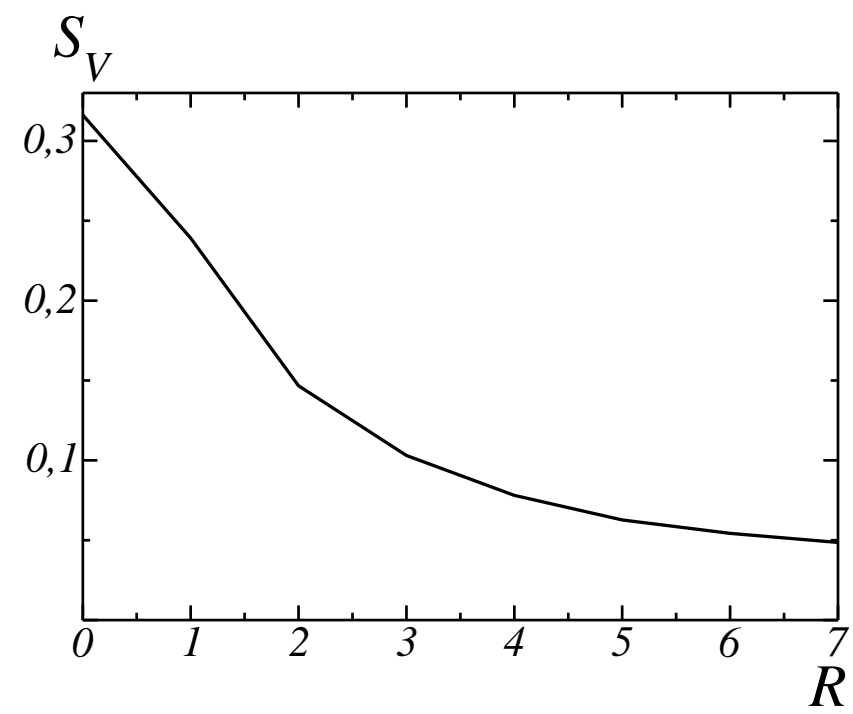

Figure 3: Fine aggregates specific surface area $S_{V}$ vs. filter size $R$. This curve is used to determine the optimal filter size, obtained when the specific surface area is close to stabilization. 


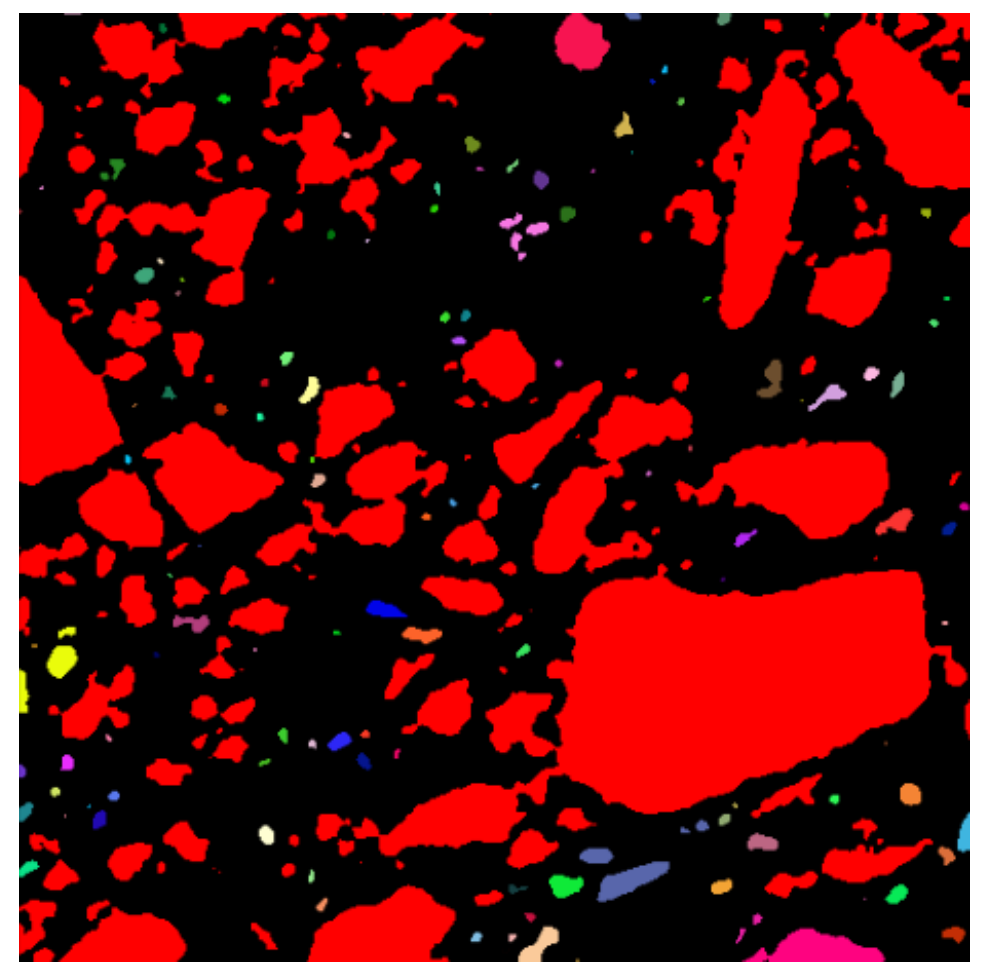

Figure 4: Image labeling of the segmented microstructure before disconnection of aggregates. Each color represents a connected component of the aggregates phase. Most aggregates are connected through a large percolating cluster (shown in red). In reality, aggregates are disconnected. 


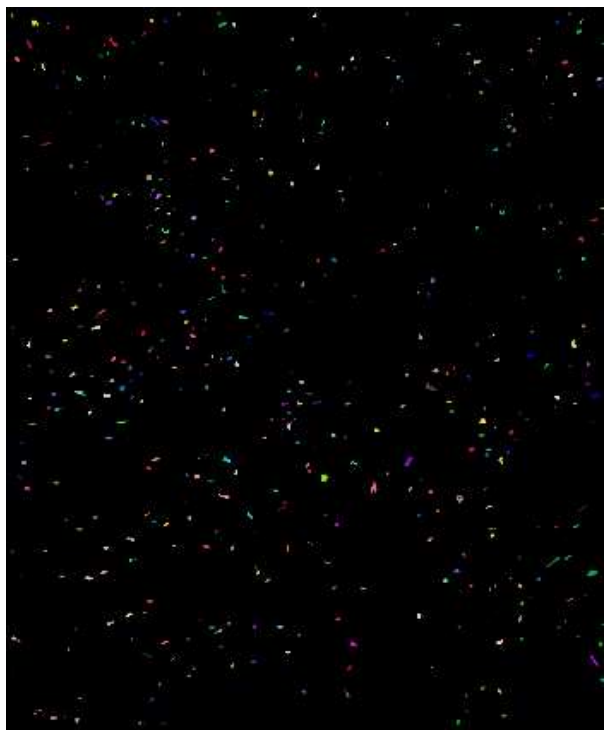

(a)

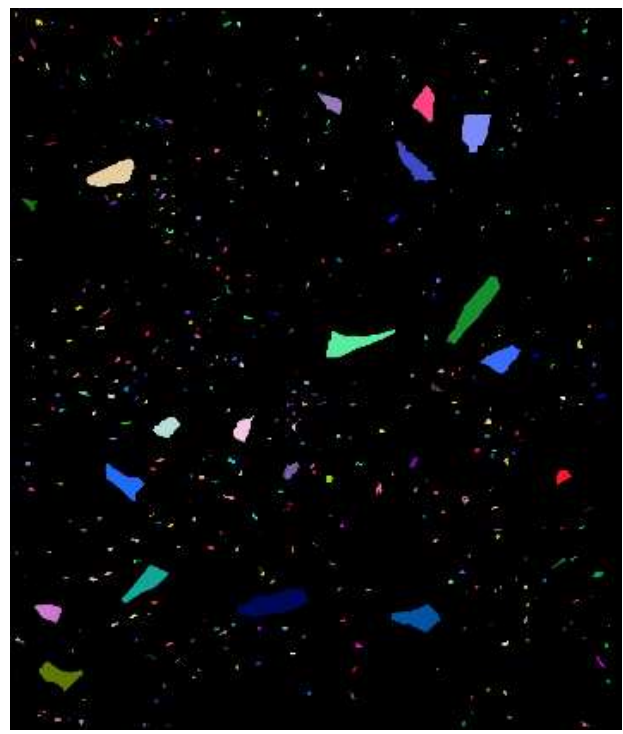

(c)

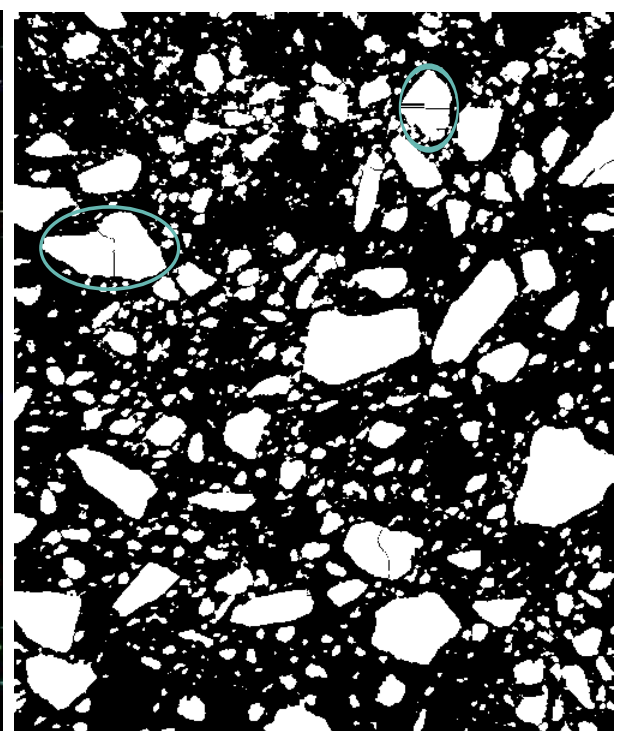

(b)

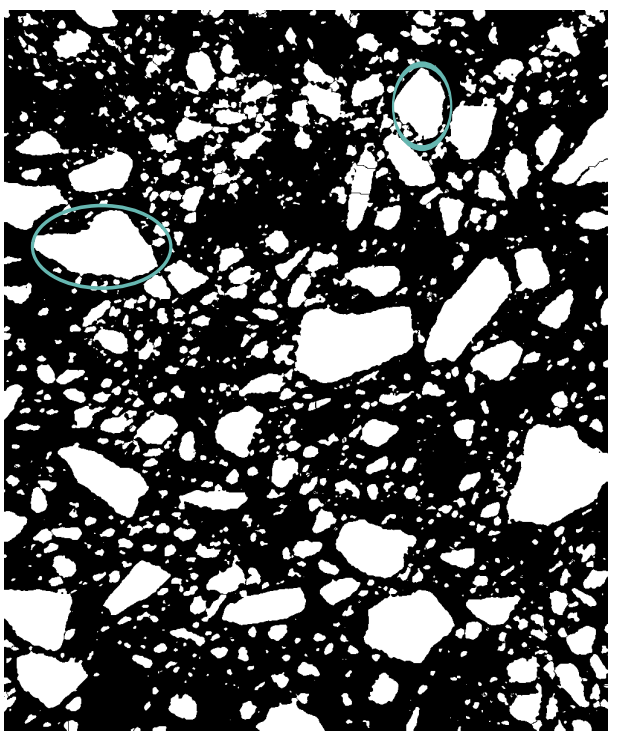

(d)

Figure 5: Classical (one-scale) (a)-(b) and multi-scale (c)-(d) aggregates disconnection using watershed: markers (a), (c), and the resulting segmentation (b), (d). With the classical one-scale segmentation, aggregates are over-segmented (b). This drawback is overcame (in b) using markers of different scales (c). Some examples of corrected oversegmentations are circled. 


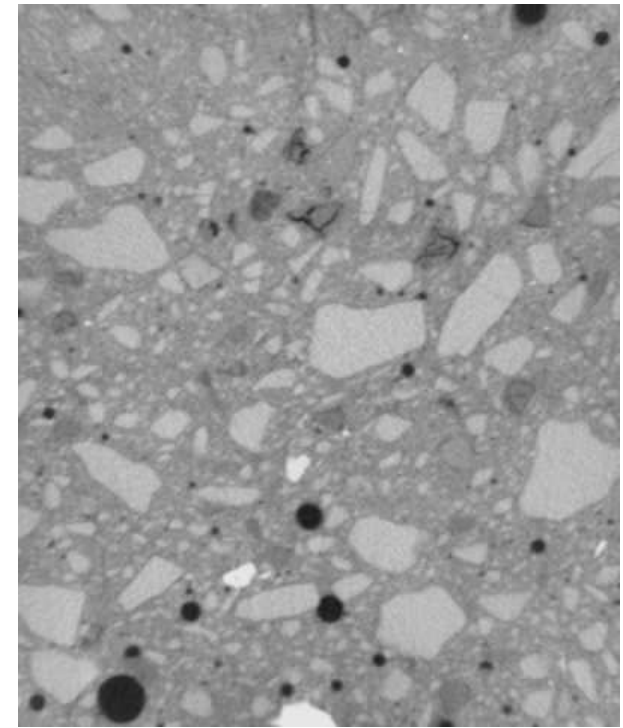

(a) Image after luminosity correction and noise removing.

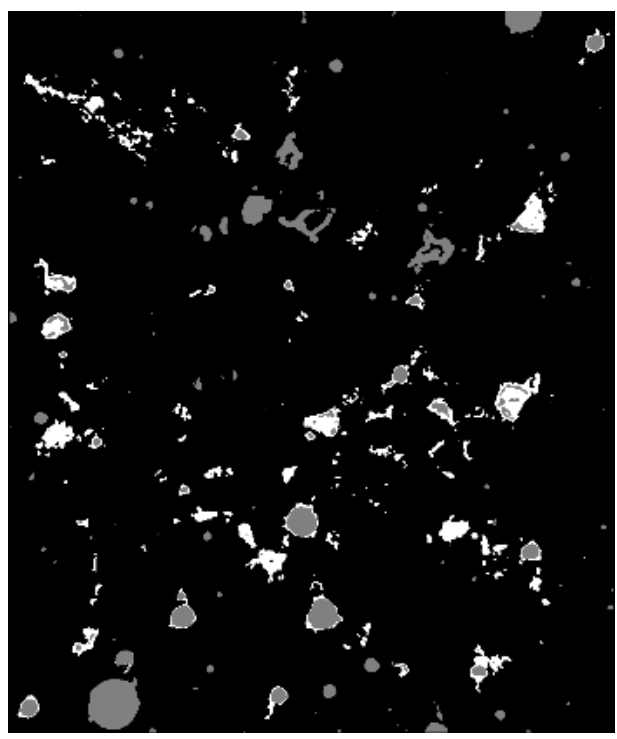

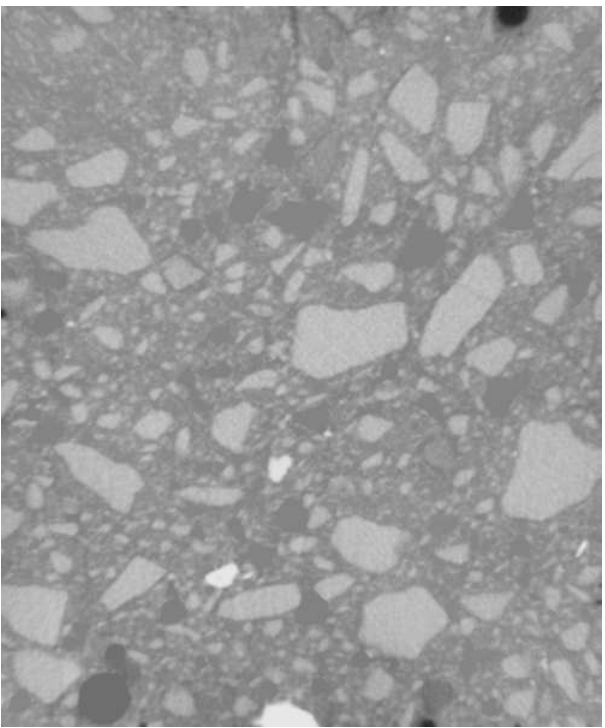

(b) Holes filling algorithm, from (a).

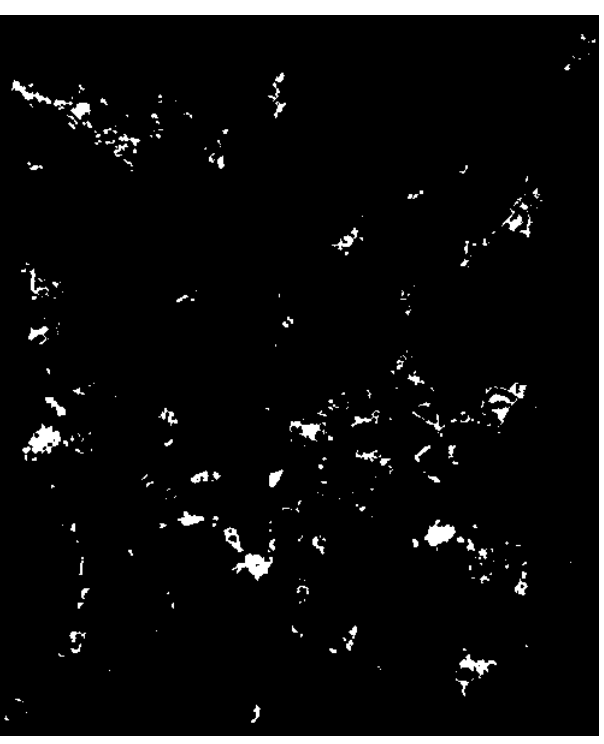

(c) From (b), selection of flat zones (d) Removing of pores and zones around (white) by volume thresholding. For pores, using dilation, from (c). comparison, pores are shown in gray. 


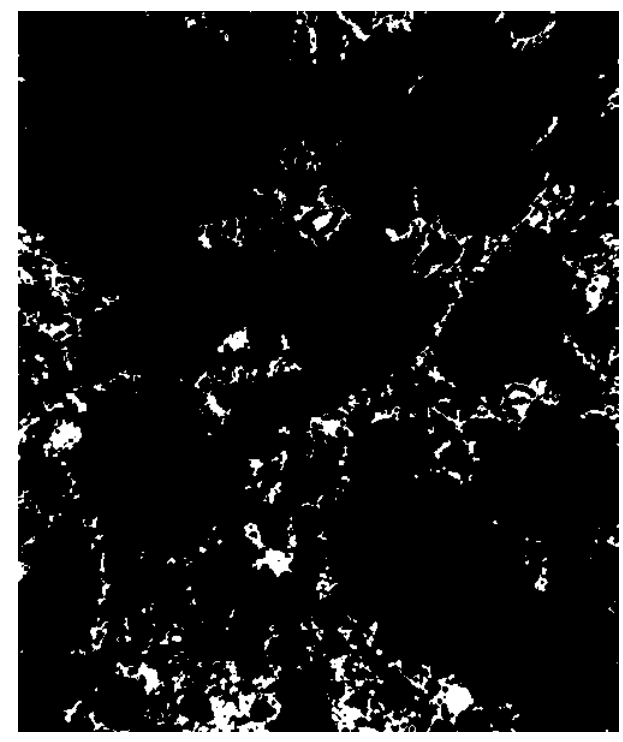

(e) Same as (d), but using thresholding of (b) instead of the treatment in (c).

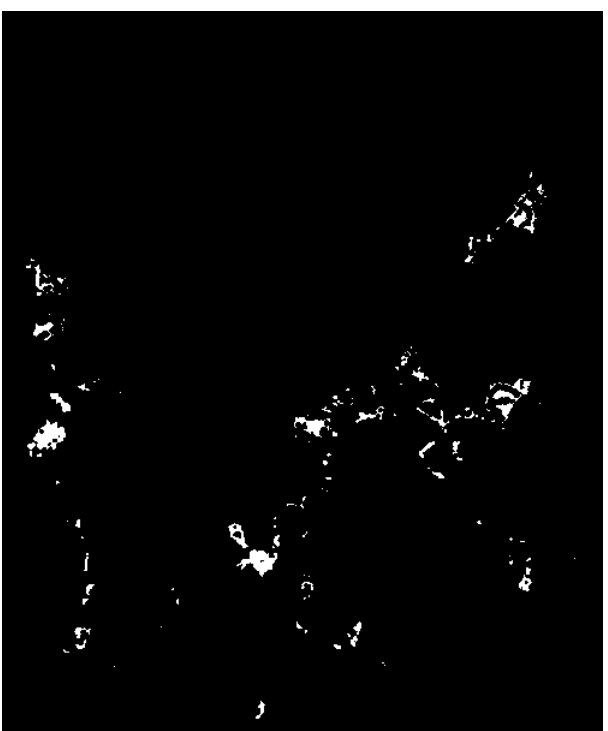

(f) Intersection of (d) and (e)

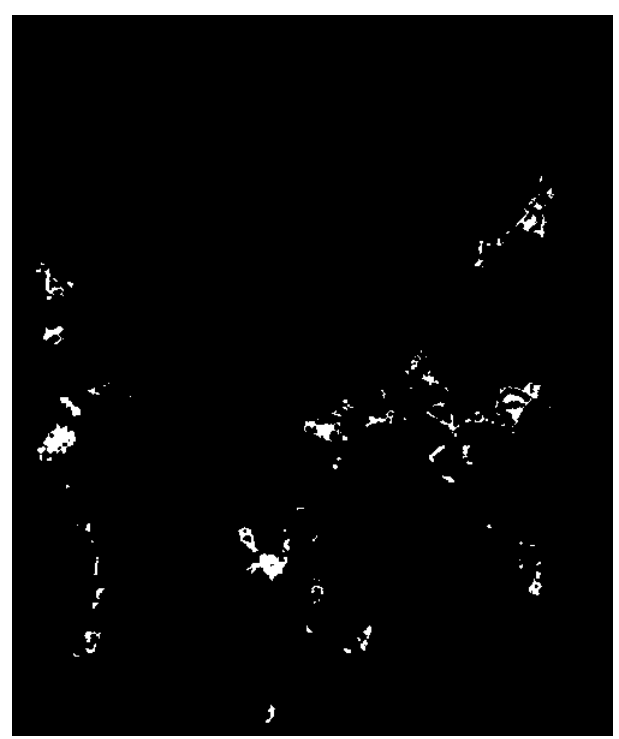

(g) Build opening, to remove small particles

Figure 6: Steps used to perform dark aggregates segmentation. The result of the holes filling algorithm in (b) is used in steps (c) and (e). 


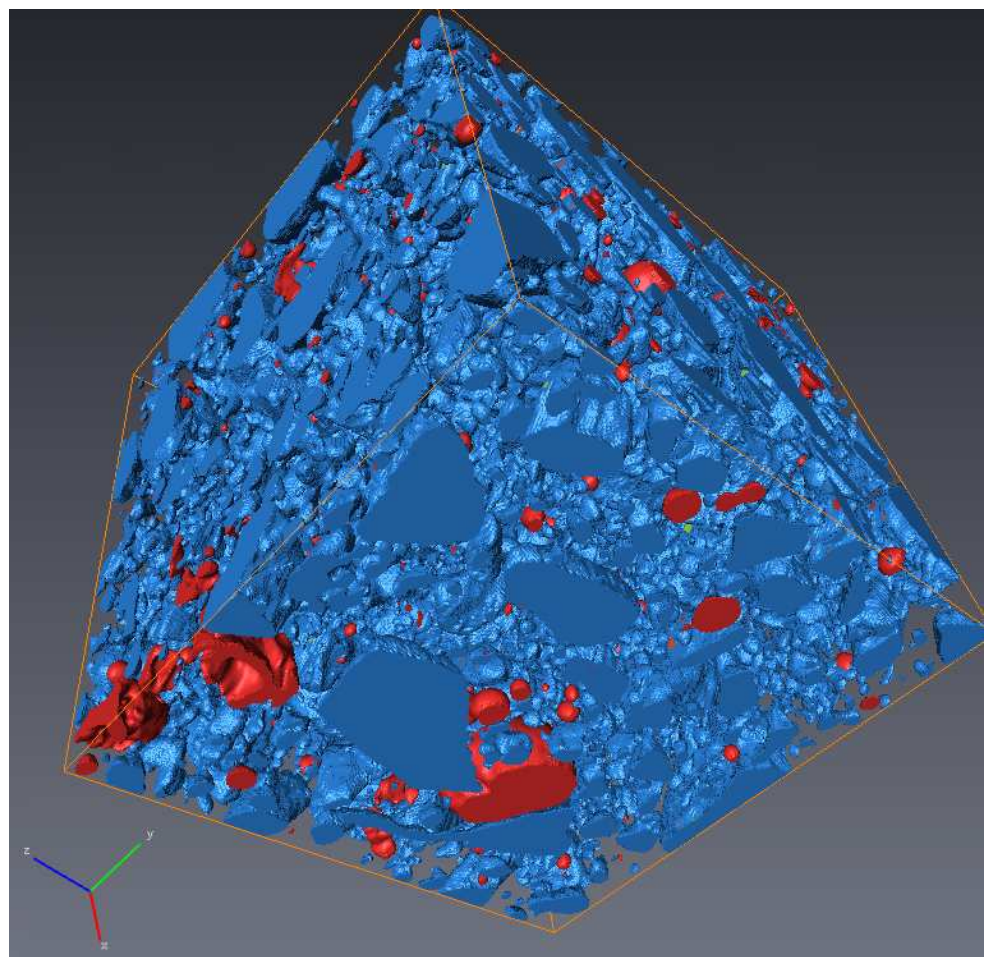

Figure 7: 3D microstructure: fine aggregates (blue) and voids (red). The matrix is transparent. The displayed volume is of size $1.25 \times 1.25 \times 1.25 \mathrm{~cm}^{3}$, the image has been subsampled to a resolution of $31 \mu \mathrm{m} /$ voxel to do the mesh. 


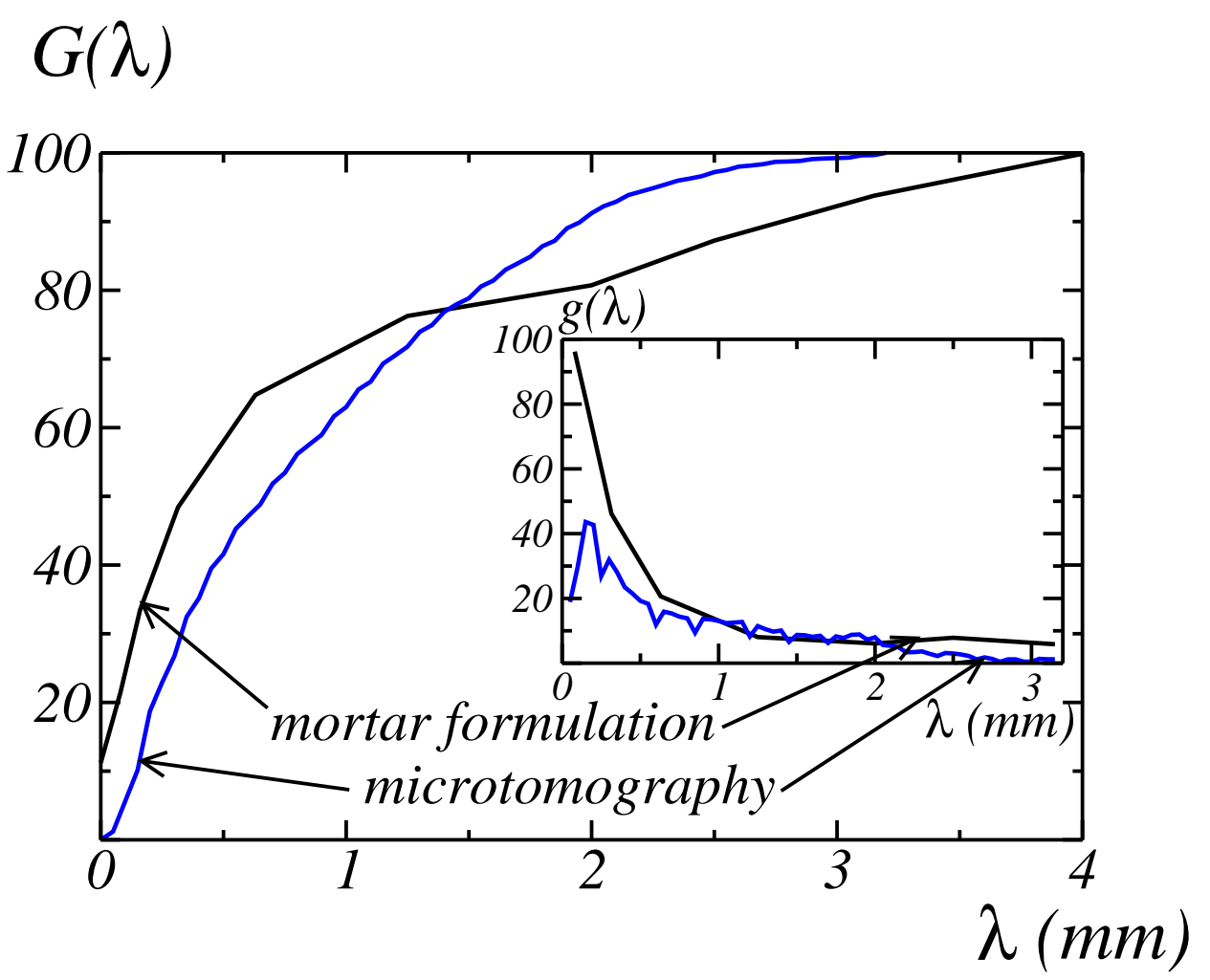

Figure 8: Cumulative fine aggregates size distribution $G$ as a function of the diameter $\lambda$. The formulation of the mortar as well as the distribution deduced from the segmentation of the microtomography image are given. The fine aggregates size distribution $g$, which takes into account the volume fractions in the sample, is also given. 


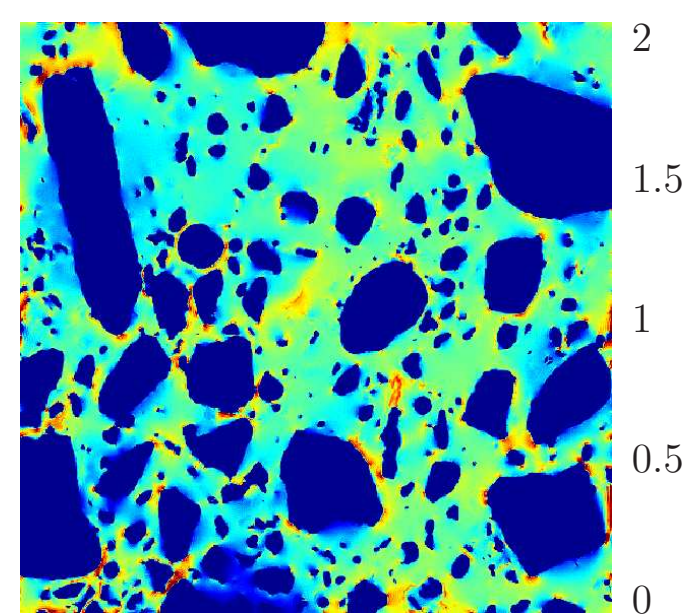

(a) $\chi=10^{-8}$

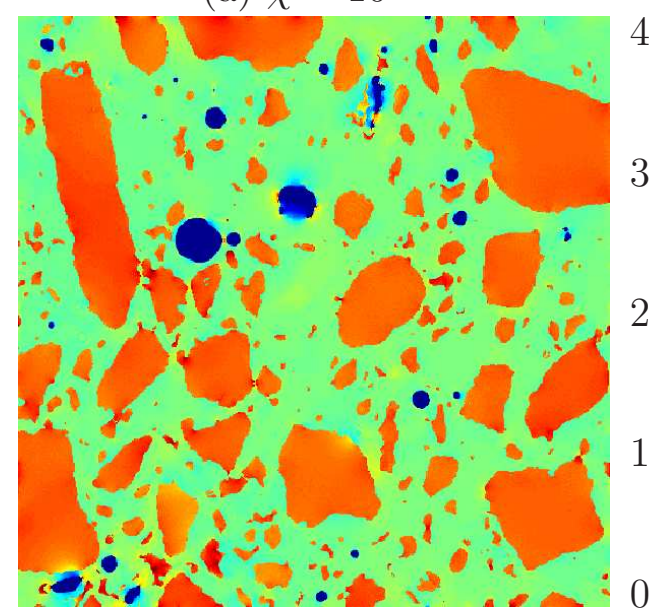

(c) $\chi=3$

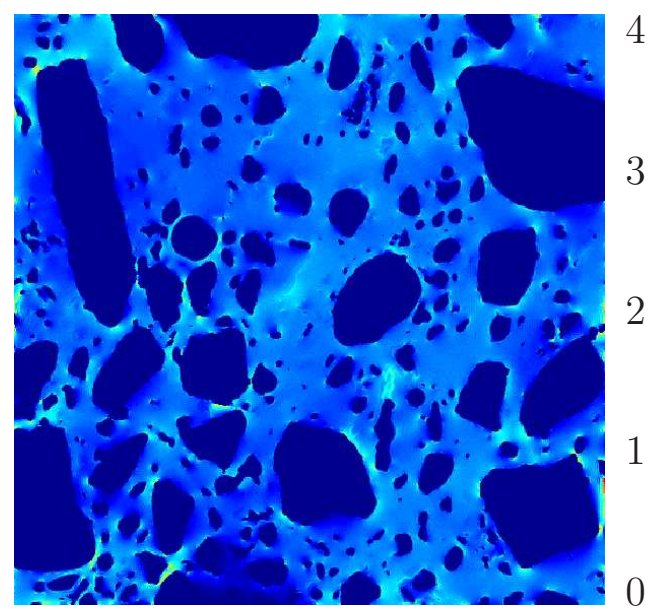

(b) $\chi=10^{-8}$

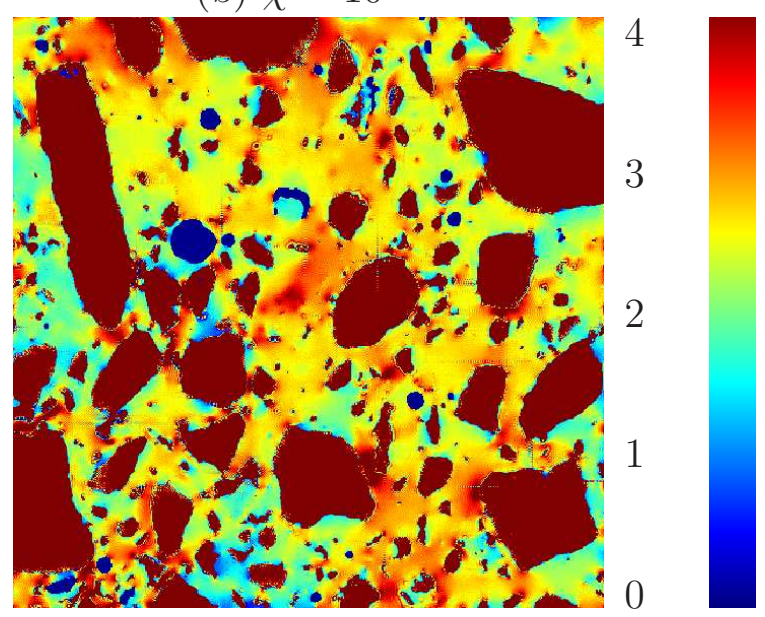

(d) $\chi=10^{2}$ 


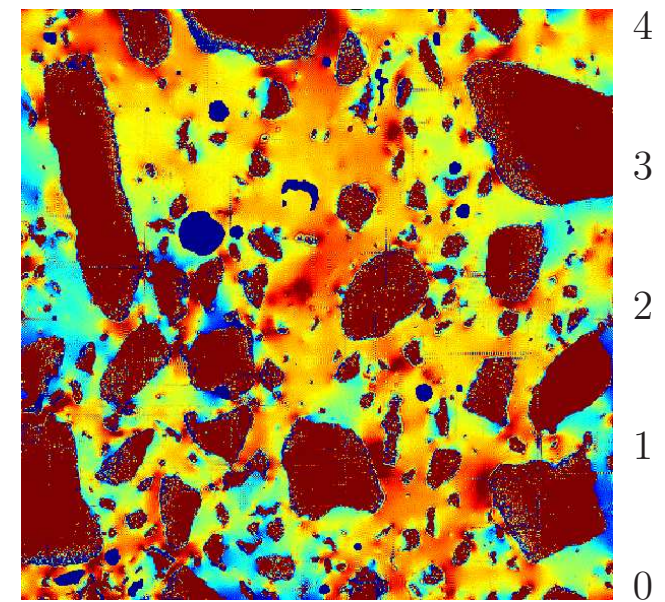

(e) $\chi=10^{3}$

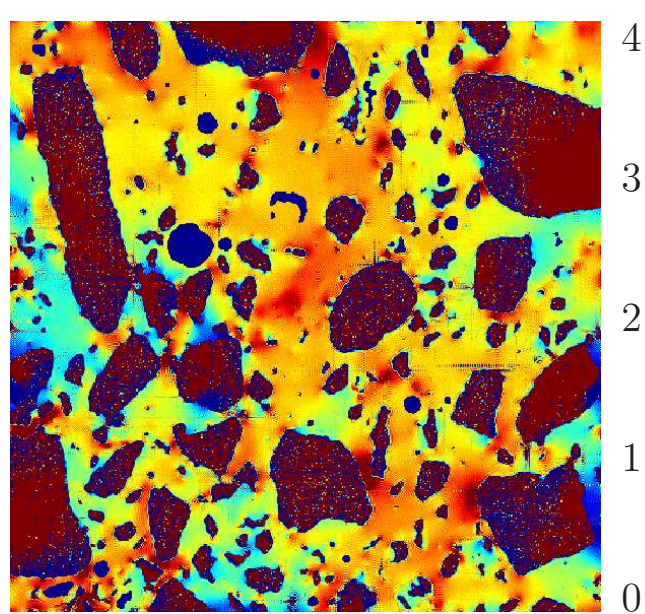

(f) $\chi=10^{4}$

Figure 9: 2D sections of the normalized mean stress component $\sigma_{m} /\left[E^{(m)}\left\langle\varepsilon_{m}\right\rangle\right]$ at varying contrasts $\chi=E^{(a)} / E^{(m)}$. The field maps are thresholded according to the color-scale as shown on the right. Hydrostatic strain loading is applied with $\left\langle\varepsilon_{m}\right\rangle=\varepsilon_{0}$ (colors online).The 3D image is of size $1.25 \times 1.25 \times 1.25 \mathrm{~cm}^{3}$ and of resolution $25 \mu \mathrm{m} /$ voxel. 

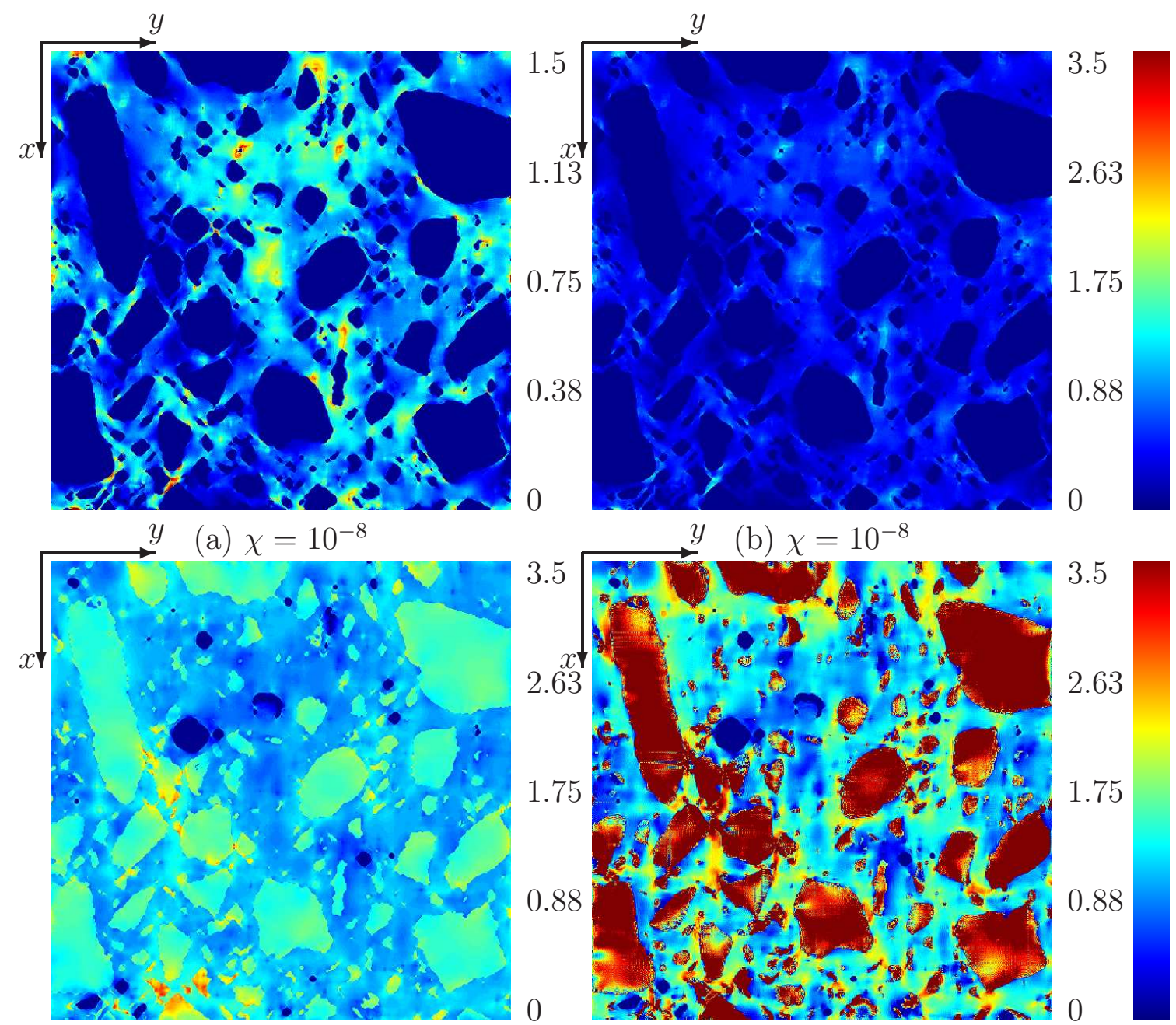

(c) $\chi=3$

(d) $\chi=10^{2}$ 


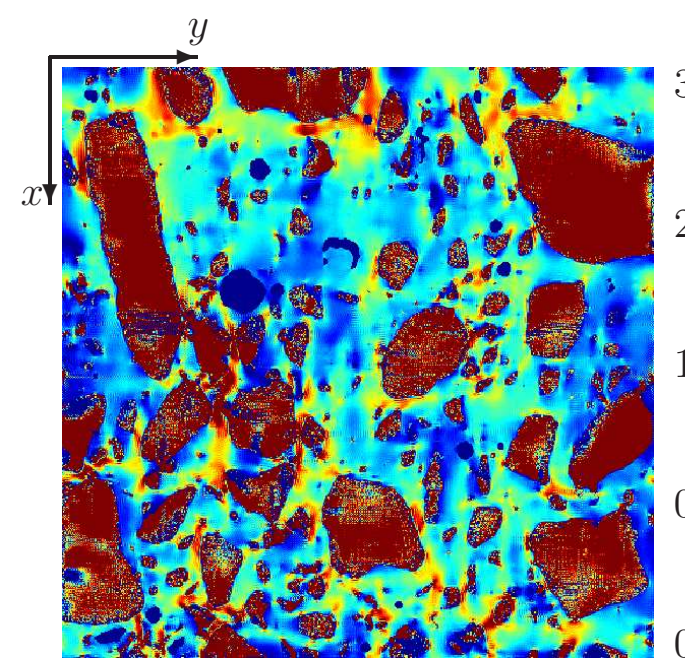

(e) $\chi=10^{3}$

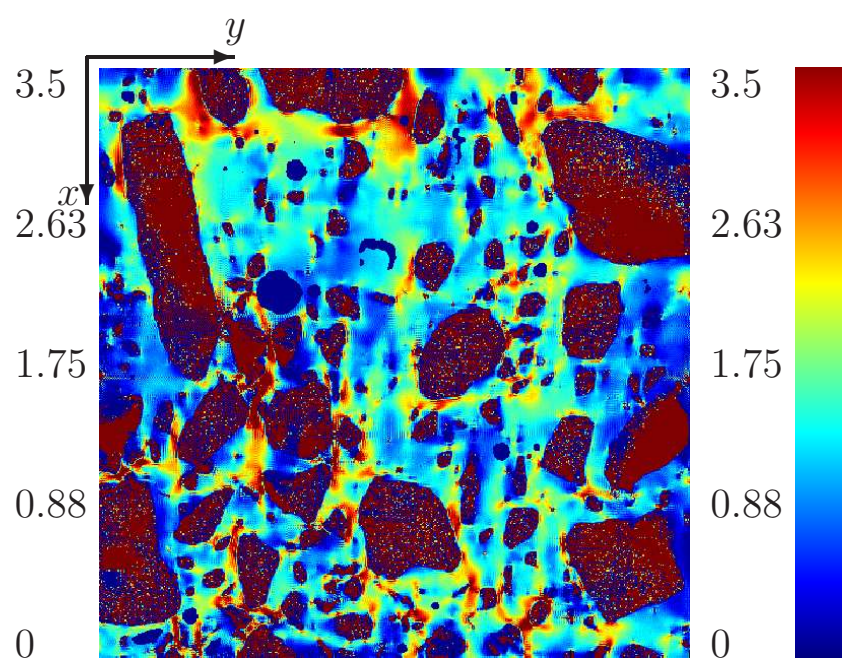

(f) $\chi=10^{4}$

Figure 10: 2D sections of the normalized shear stress component $\sigma_{x y} /\left[E^{(m)}\left\langle\varepsilon_{x y}\right\rangle\right]$ at varying contrasts $\chi=E^{(a)} / E^{(m)}$. The field maps are thresholded according to the color-scale as shown on the right. Shear strain loading is applied with $\left\langle\varepsilon_{x y}\right\rangle=\varepsilon_{0}$ (colors on-line). The 3D image is of size $1.25 \times 1.25 \times 1.25 \mathrm{~cm}^{3}$ and of resolution $25 \mu \mathrm{m} /$ voxel. 


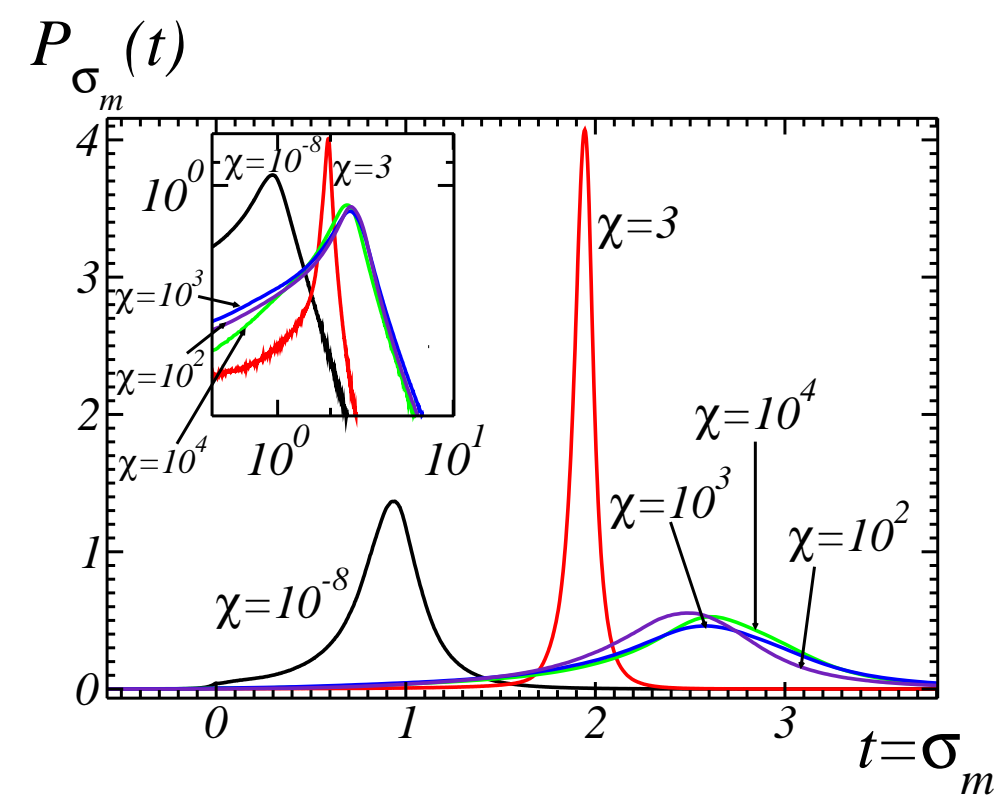

(a) Hydrostatic strain loading

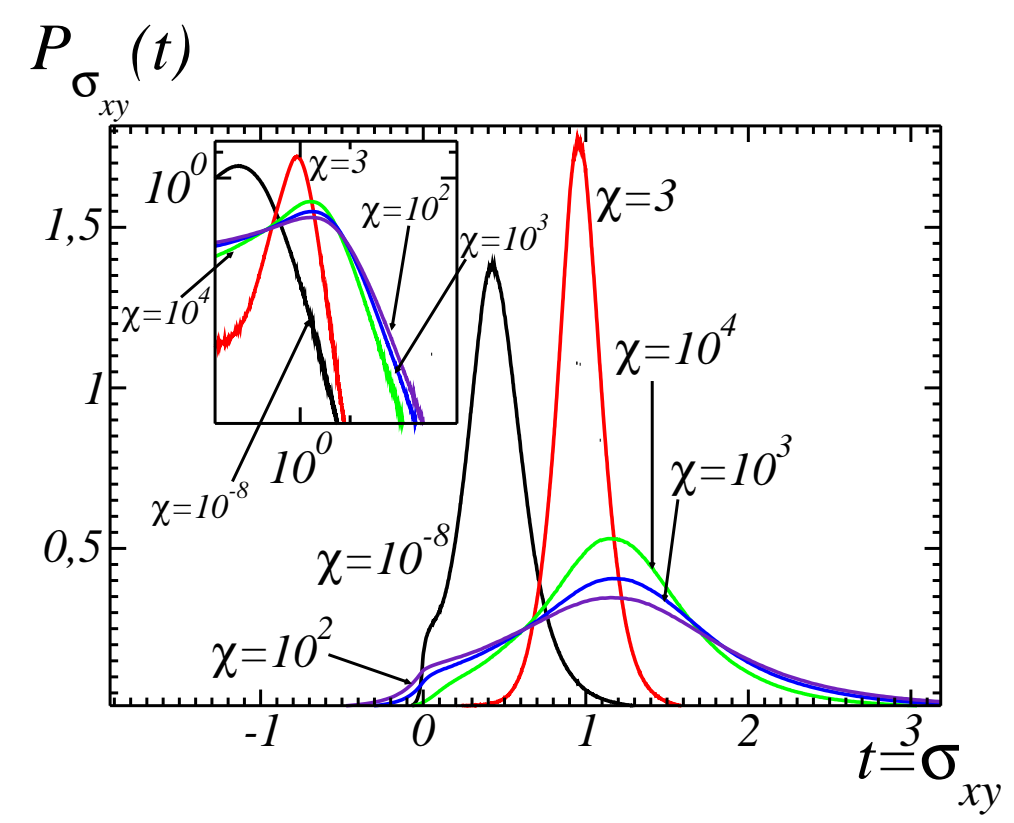

(b) Shear strain loading

Figure 11: Field histograms $P_{\sigma_{m}}(t)$ and $P_{\sigma_{x y}}(t)$, i.e. probability density values, of the mean stress field $\sigma_{m}$ and the shear stress component field $\sigma_{x y}$, at varying contrasts $\chi=$ $E^{(a)} / E^{(m)}=10^{-8}, 3,100,1000,10^{4}$. Distribution tails are also given, using a $\log -\log$ scale. 


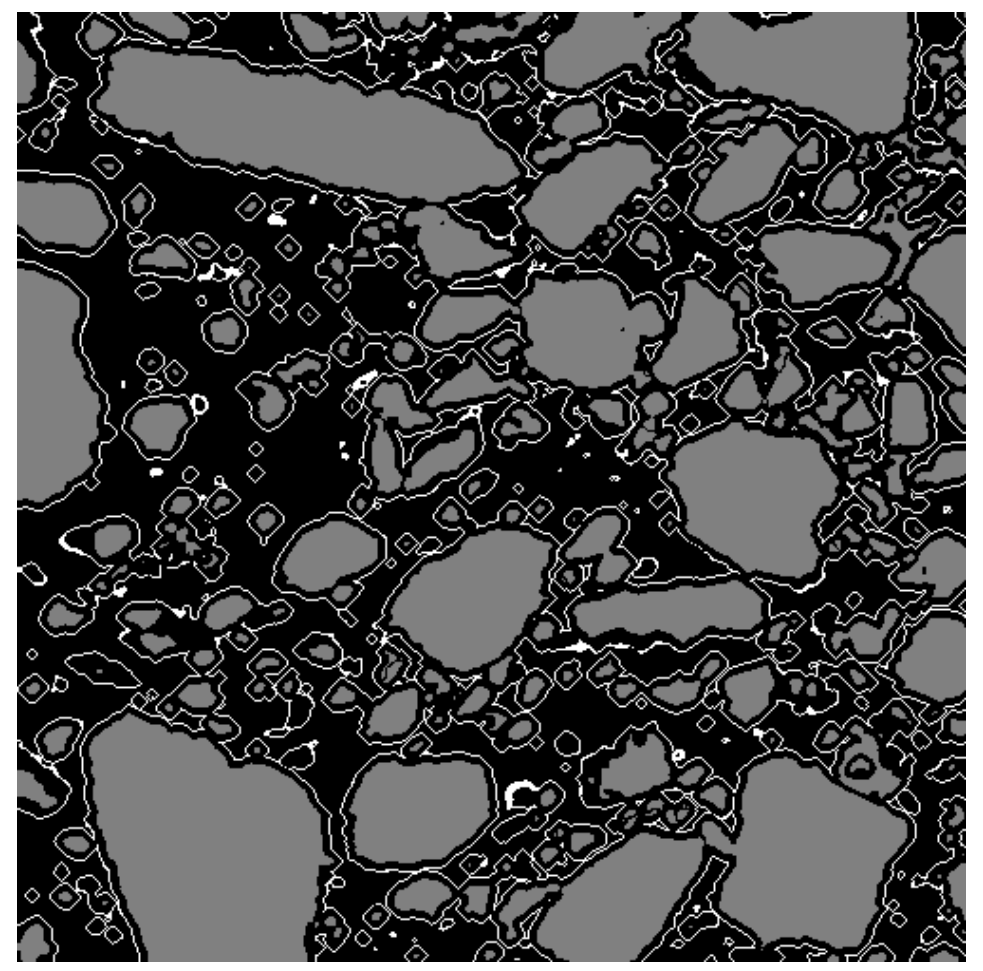

Figure 12: 2D section of sets used to evaluate the mean $m_{a}(r)$ of a field in the matrix at a distance $r=5$ of fine aggregates. Fine aggregates are given in gray and the set $\left.\left.X_{r}=\left(A_{j} \oplus B(r)\right)\right) \cap\left(A_{j} \oplus B(r-1)\right)\right)^{c} \cap A_{m}$ is given in white. 


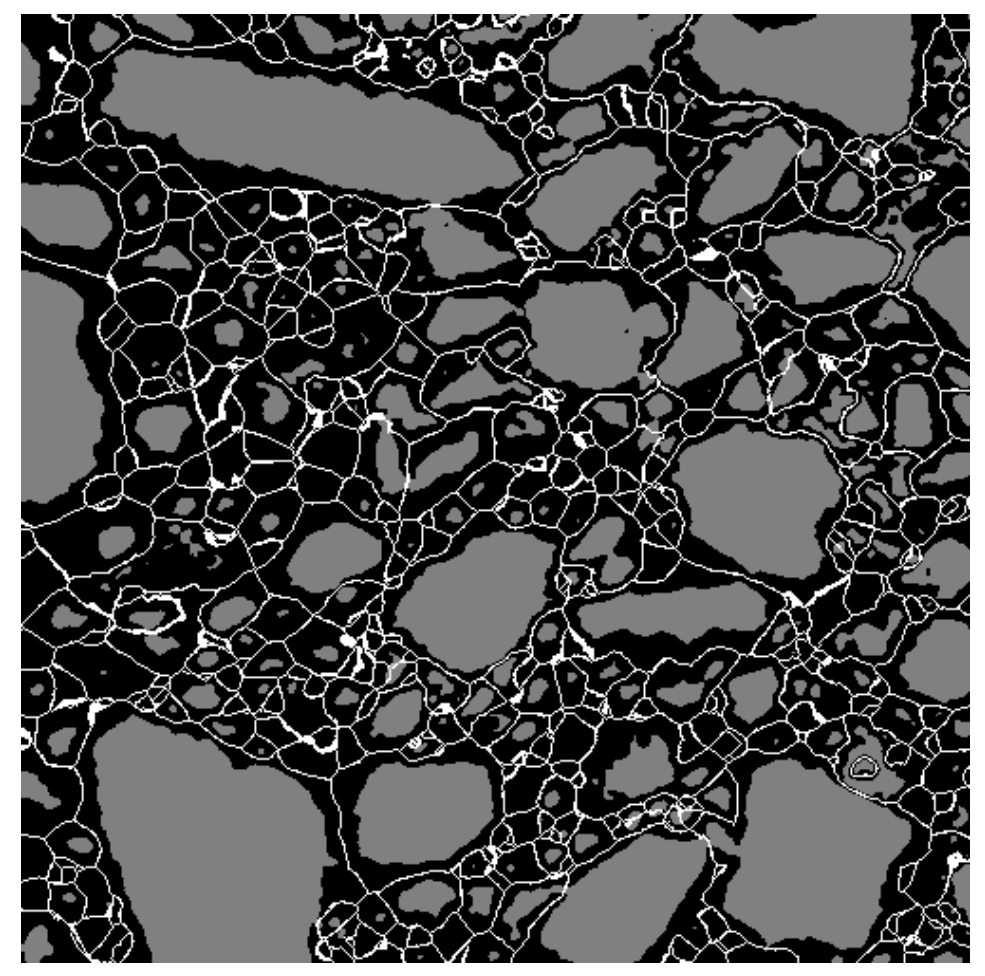

Figure 13: 2D section of the skeleton by influence zone (SKIZ) of the fine aggregates phase. Fines aggregates are given in gray and the SKIZ is given in white. 


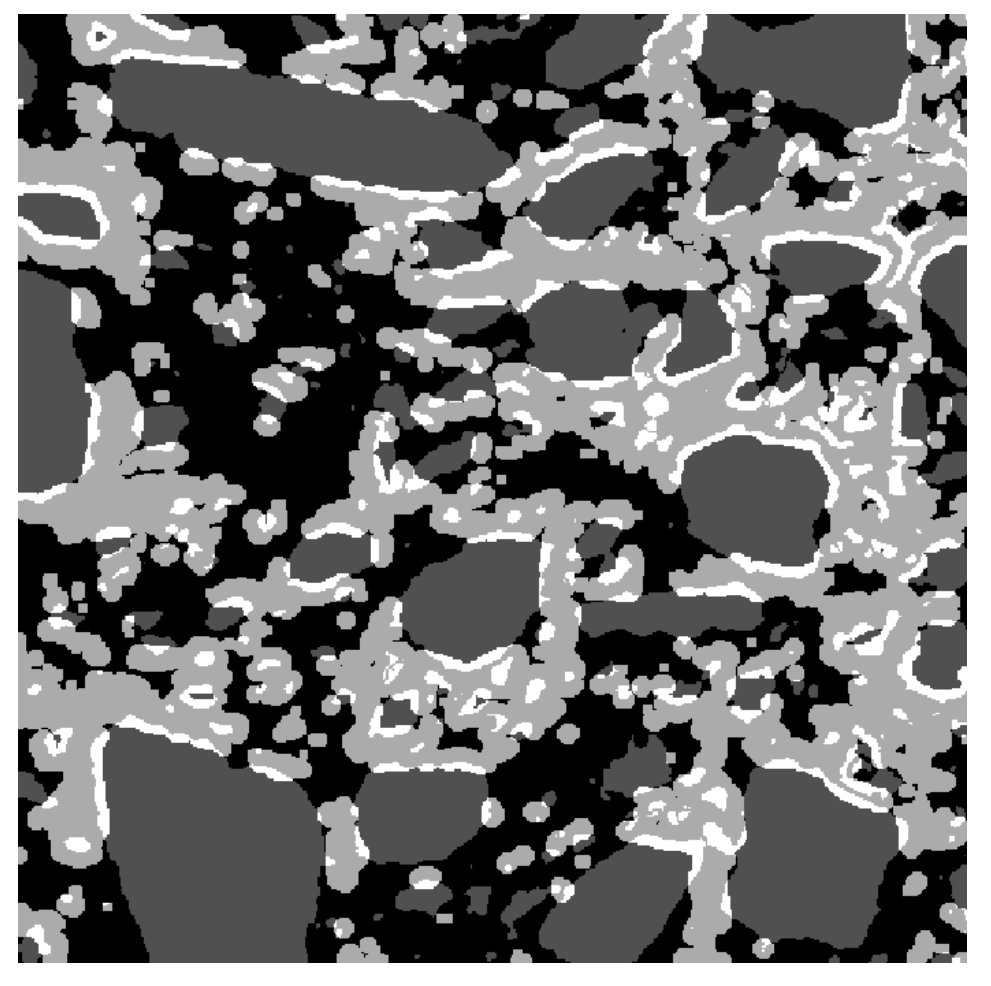

Figure 14: 2D section of the sets used to evaluate the function $\rho_{a}(r)$ at $r=5$. Aggregate phase (set $A_{j}$ ) is given in dark gray, $A_{k} \oplus B(r)$ ( $A_{k}$ corresponds to regions of high values of the considered stress field component), is given in light gray and their intersection $A_{j} \cap\left(A_{k} \oplus B(r)\right)$ is given in white. 


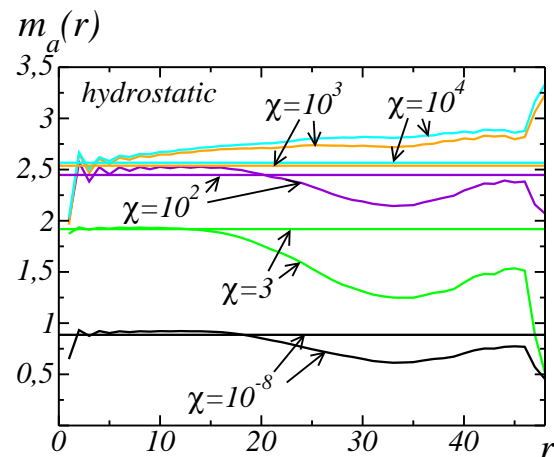

(a)

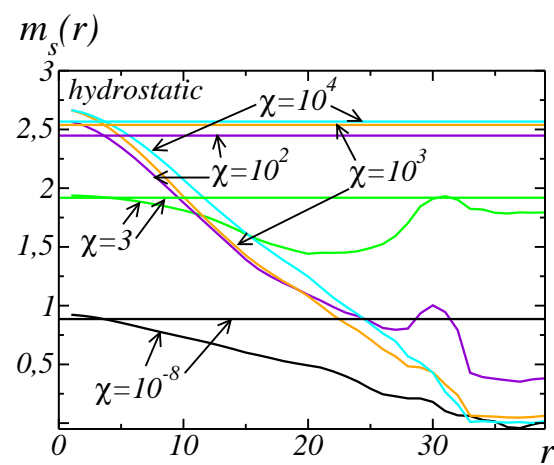

(c)

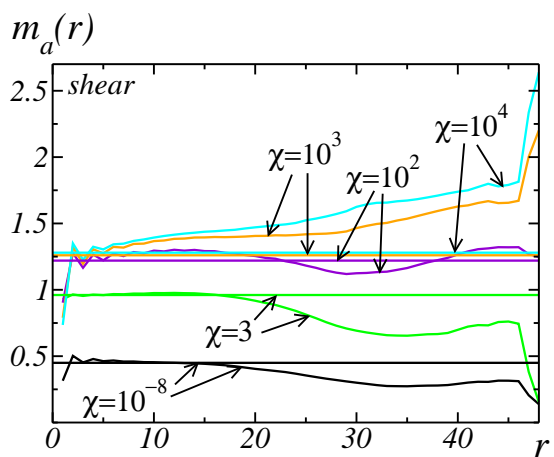

(b)

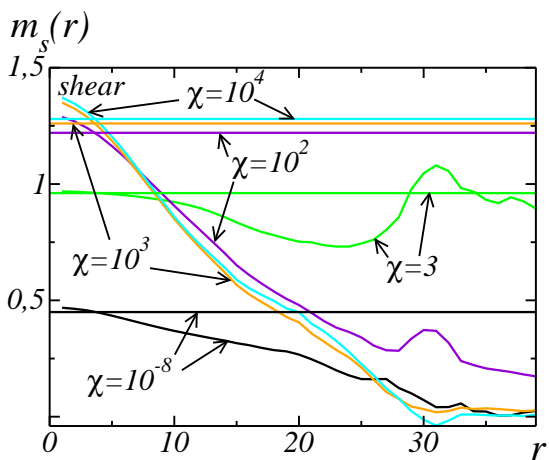

(d)

Figure 15: Mean $m_{a, s}(r)$ of the parallel stress component $\sigma_{m}$ or $\sigma_{x y}$ as a function of the distance $r$ at various aggregates/matrix contrasts $\chi$. The distance $r$ is calculated from the aggregates (a, b) or from the aggregates SKIZ (c, d) (see Sec. 6.1). Hydrostatic or shear strain loading is applied ( $\mathrm{a}, \mathrm{c}$ and b, d, resp.). At each contrast, a straight horizontal line indicates the mean stress component in the matrix. 


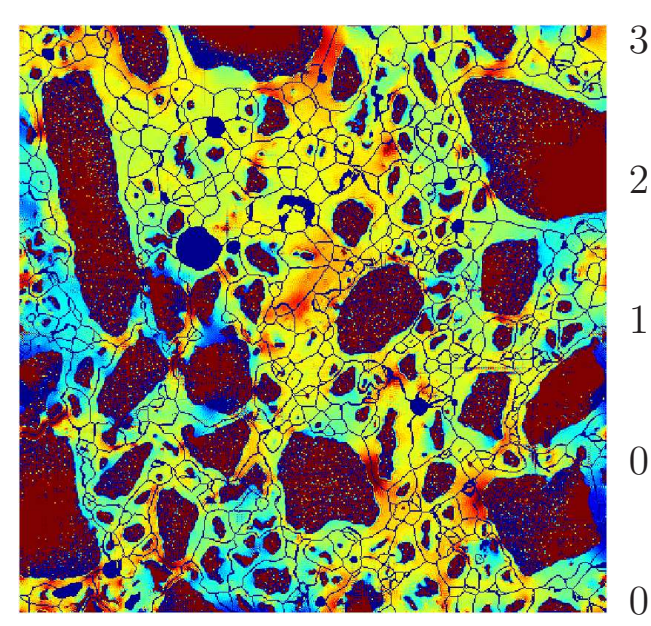

(a)

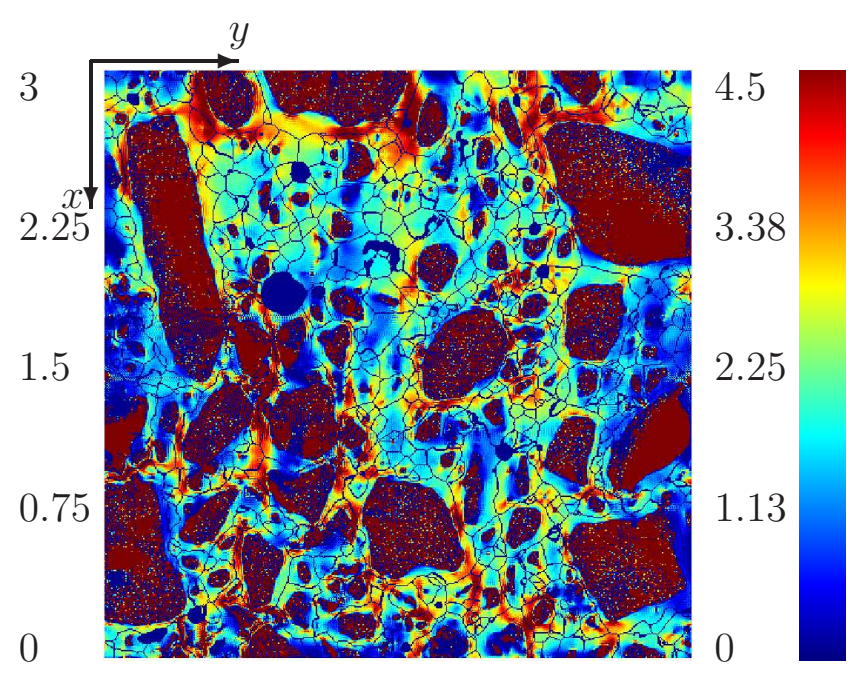

(b)

Figure 16: $2 \mathrm{D}$ cut at $z=$ cst of the parallel stress component $\sigma_{m}$ and $\sigma_{x y}$ (maps a and b, resp.) with applied hydrostatic or shear strain loading, resp., with aggregates/matrix contrast $\chi=10^{4}$. The stress field values correspond to the color scale at right (values outside of the range given at the right of each map are thresholded). The aggregates SKIZ is superimposed on the maps and shown in blue. The $3 \mathrm{D}$ image is of size $2.5 \times 3 \times 2.5 \mathrm{~cm}^{3}$ and of resolution $25 \mu \mathrm{m} /$ voxel. 


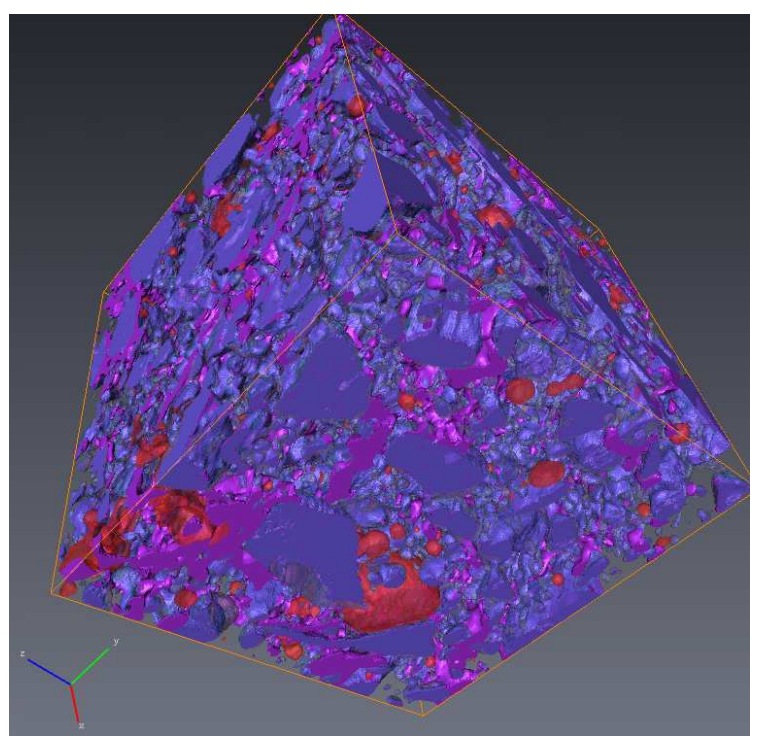

(a) Hydrostatic strain loading

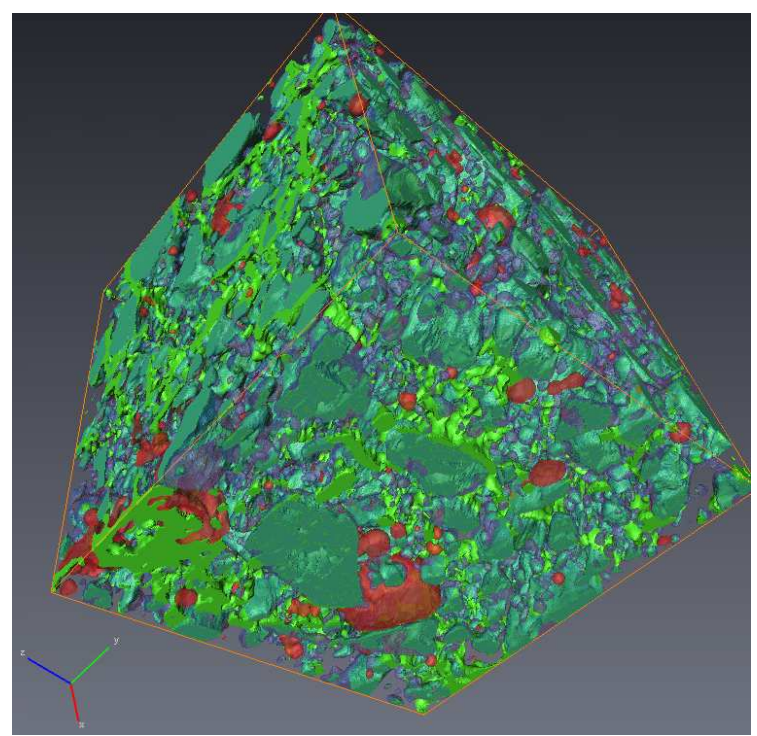

(b) Shear strain loading

Figure 17: 3D view of the microstructure (fine aggregates in blue, voids in red and transparent matrix), and of thresholded stress field component $\sigma_{m}$ or $\sigma_{x y}$ for hydrostatic strain loading or shear strain loading respectively, in purple and green respectively. The displayed volume is of size $1.25 \times 1.25 \times 1.25 \mathrm{~cm}^{3}$, the image has been subsampled to a resolution of $31 \mu \mathrm{m} /$ voxel to do the mesh. 


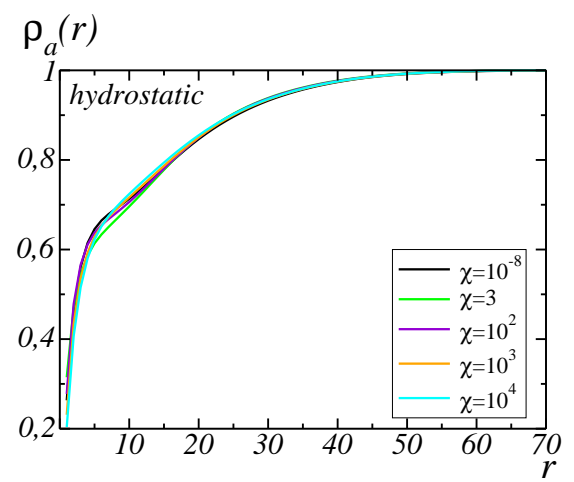

(a)

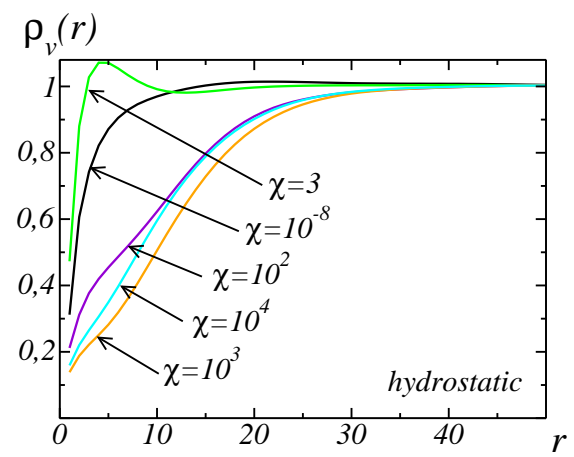

(c)

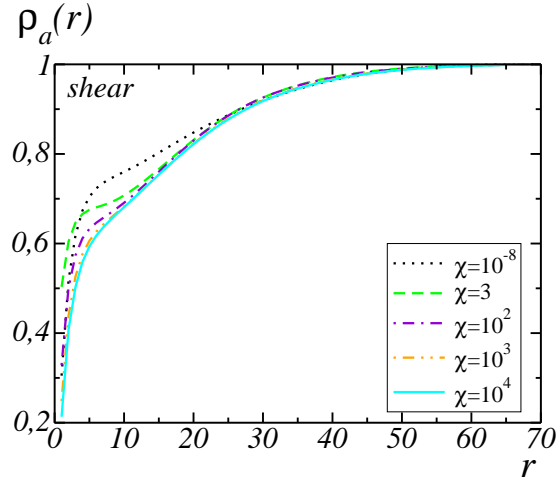

(b)

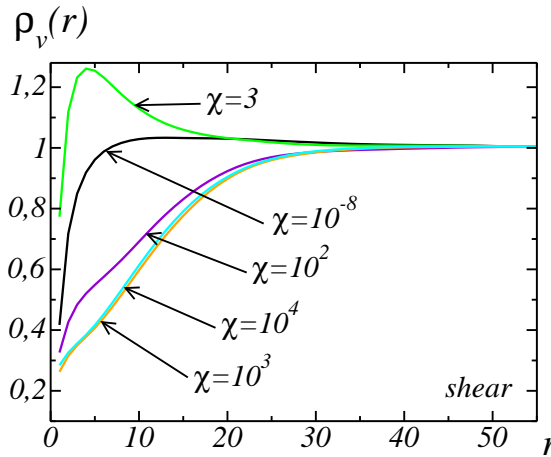

(d)

Figure 18: Function $\rho_{a, v}(r)$ used to measure the correlation between one of the material phase (a, b: fine aggregates, c, d: voids) and regions of high-stress. Hydrostatic or shear strain loading is applied ( $\mathrm{a}, \mathrm{c}$ and b, d, resp.). High-stress regions are determined by thresholding the parallel stress component $\sigma_{m}$ or $\sigma_{x y}$. 